\title{
La possible modification de la procédure de révision \\ de la Constitution belge
}

\section{I - INTRODUCTION}

1. - Le 4 avril 2003, le Parlement fédéral belge ${ }^{1}$ a voté une déclaration de révision de la Constitution permettant de modifier l'article 195 de la Constitution belge, qui règle la procédure de révision constitutionnelle. La publication de cette déclaration ${ }^{2}$ au Moniteur belge du 10 avril a entraîné la dissolution de plein droit des Assemblées législatives et la tenue d'élections fédérales qui ont eu lieu le 18 mai $^{3}$.

2. - La procédure de révision constitutionnelle figure parmi les normes les plus importantes d'un ordre juridique donné. C'est elle qui fixe les conditions dans lesquelles les règles situées au sommet de la hiérarchie des normes juridiques peuvent être modifiées ${ }^{4}$.

A ce titre, la perspective de voir l'ordre juridique belge changer sa procédure de révision constitutionnelle retiendra certainement l'attention du constitutionnaliste français ${ }^{5}$. Il en ira a fortiori ainsi pour deux raisons.

D’une part, la Belgique n'a jamais changé de Constitution depuis son indépendance en 1830. En effet, malgré une opération de coordination en $1994^{6}$, la Loi fondamentale belge actuellement en vigueur date de $1831^{7}$, ce qui en fait l'une des plus vieilles Constitutions au monde encore en vigueur.

\footnotetext{
${ }^{1}$ Composé de deux Assemblées, à savoir de la Chambre des Représentants et du Sénat.

${ }^{2}$ A strictement parler, il existe deux déclarations de révision distinctes (mais identiques), l'une émanant des deux Assemblées, l'autre du Roi (c'està-dire du Gouvernement fédéral). Elles sont cependant toujours publiées simultanément au Moniteur belge, le Journal officiel belge. Lorsqu'elles ne sont pas adoptées le même jour, on s'accorde généralement à retenir la date de la déclaration de révision émanant du Roi (signée en l'occurrence le 9 avril 2003).

${ }^{3}$ Voy. le Communiqué du 10 avril 2003 portant convocation des électeurs aux élections fédérales, Moniteur belge, 15 avril, et l'Arrêté royal du 10 avril 2003 réglant certaines opérations pour l'élection des Chambres législatives fédérales du 18 mai 2003, Moniteur belge, 18 avril, $2^{2}$ édition.

${ }^{4}$ Pour une approche théorique et générale de la notion de révision de la Constitution, voy. notamment O. Pfersmann, «L'élaboration et la révision des Constitutions : la production du droit constitutionnel formel », in : L. Favoreu et autres, Droit constitutionnel, $3^{\mathrm{e}}$ édition, Paris, Dalloz, $2000, \mathrm{p}$. 119-139.

${ }^{5}$ Nous nous permettons de renvoyer le lecteur désireux d'approfondir l'étude du présent sujet à la contribution publiée dans les Actualités du Droit, Revue de la Faculté de Droit de Liège, Editions Kluwer, 2002, p. 403-442.

${ }^{6}$ Par application de l'article 132 de la Constitution belge du 7 février 1831, la numérotation des articles de cette Constitution été modifiée le 17 février 1994 (Moniteur belge, 17 février). Par la même occasion, certaines dispositions constitutionnelles ont subi de légères adaptations terminologiques. Cette opération dite de «coordination » ne constitue cependant qu'une simple intervention du Constituant dérivé et n'affecte pas la continuité dans l'application des dispositions de 1831. Dès lors, si la Constitution ainsi remaniée est généralement désignée par les termes «la Constitution coordonnée du 17 février 1994 », il n'en demeure pas moins que le Constituant originaire belge n'est plus intervenu depuis 1831 et que, par conséquent, la Constitution belge continue, d'un point de vue normatif, à dater de 1831 .

${ }^{7}$ Elle a été solennellement proclamée le 7 février 1831, promulguée le 11 février, et est entrée en vigueur le 25 février de la même année. Publication en a été faite au Bulletin officiel des décrets du Congrès national de la Belgique et des arrêtés du Pouvoir exécutif, année 1831, n $14, \mathrm{p}$. 160-178.
} 
D'autre part, la Belgique n'a jamais changé de procédure de révision constitutionnelle. C'est ainsi que l'article 195 de la Constitution - disposition qui avant la coordination de 1994 portait le numéro $131^{8}$ dispose de manière inchangée depuis le 7 février 1831 :

'Le pouvoir législatif fédéral a le droit de déclarer qu'il y a lieu à la révision de telle disposition constitutionnelle qu'il désigne.

Après cette déclaration, les deux Chambres sont dissoutes de plein droit.

II en sera convoqué deux nouvelles, conformément à l'article 46.

Ces Chambres statuent, d'un commun accord avec le Roi, sur les points soumis à révision.

Dans ce cas, les Chambres ne pourront délibérer si deux tiers au moins des membres qui composent chacune d'elles ne sont présents; et nul changement ne sera adopté s'il ne réunit au moins les deux tiers des suffrages.

3. - Au vu de cette déclaration du 9 avril 2003, il semble opportun de prolonger, avant même le dépôt au Parlement fédéral des premières propositions visant à modifier l'article 195, le débat doctrinal presque centenaire sur cette question ${ }^{9}{ }^{10}$.

Ce débat, mené par des plumes plus autorisées que la nôtre, s'est cependant avant tout intéressé aux imperfections de la version actuelle de l'article 195 et a cherché à évaluer l'opportunité de sa modification éventuelle ; l'objectif principal n'a donc pas été de se pencher sur son libellé futur.

4. - La déclaration du 9 avril 2003 a sensiblement changé la donne : on semble aujourd'hui fondé à postuler que l'article 195 de la Constitution coordonnée sera modifié sous la législature qui vient de s'ouvrir $^{11}$, ce qui pose avec acuité le problème de sa formulation nouvelle.

A cet égard, notre contribution vise avant tout à démontrer que si en théorie « [1]a boîte de pandore est ouverte $»^{12}$ pour une nouvelle formulation de l'article 195, en pratique la marge de manœuvre est beaucoup plus étroite. Il en va ainsi pour des motifs tant politiques qu'institutionnels.

\footnotetext{
8 Nous utiliserons généralement la numérotation applicable depuis 1994 (article 195). Il nous arrivera cependant d'employer l'ancienne numérotation (article 131) lors de l'analyse de documents antérieurs à la coordination de la Constitution.

${ }^{9}$ Sur le problème de l'opportunité d'une révision de l'article 195 de la Constitution belge, voy. les contributions suivantes, classées de manière chronologique: O. Orban, Le Droit constitutionnel de la Belgique, tome 2, Liège et Paris, Dessain et Giard \& Brière, 1908, p. 704-731, spécialement p. 723-724 et 727-728 ; P. Wigny, Propos constitutionnels, Bruxelles, Bruylant, 1963, spécialement p. $24-67$; A. Verhasselt-Luykx, «De rol van de preconstituante bij de grondwetsherziening », Tijdschrift voor Bestuurswetenschappen en Publiekrecht, 1971, p. 230-239; F. Delpérée, «Une crise en trois temps », Journal des Tribunaux, 1986, p. 117-130, spécialement p. 121-122 ; F. Perin, « Y a-t-il trois pouvoirs constituants ? ", Annales de Droit de Liège, 1987, p. 3-16 ; F. Delpérée, " Le processus de modification de la Constitution belge », in : La Révision de la Constitution, Journées d'études des 20 mars et 16 décembre 1992, Paris et Aix-en-Provence, Economica et PUAM, 1993, p. 67-77, spécialement p. 72-77 ; A. Alen, Belgisch Staatsrecht, Deurne, Kluwer, 1995, p. 69-70 ; J. van Nieuwenhove, « De herziening en de coördinatie van de Grondwet », in : M. van der Hulst et L. Veny (éds.), Parlementair Recht-Commentaar \& Teksten, Gand, Mys \& Breesch, mise à jour 1998, 120 p., spécialement p. 95-118; J.-Cl. Scholsem, «Brèves réflexions sur une éventuelle révision de l'article 195 de la Constitution », Revue belge de droit constitutionnel, 1999, p. 99-105; X. Delgrange et H. Dumont, « Le rythme des révisions constitutionnelles et l'hypothèse de l'accélération du temps juridique », in: P. Gérard, F. Ost et M. van de Kerchove (dirs.), L'accélération du temps juridique, Bruxelles, Publications des Facultés universitaires Saint-Louis, 2000, p. 413-468 ; M. Uyttendaele, Précis de Droit constitutionnel belge, Bruxelles, Bruylant, 2001, p. 103-112. - En raison de son caractère particulièrement approfondi, la contribution prémentionnée de J. van Nieuwenhove est digne d'une attention spéciale. De plus amples références peuvent être trouvées dans la contribution mentionnée à la note 5, supra.

${ }^{10}$ Sur la révision de la Constitution belge en général, mais sans traiter du problème de l'opportunité d'une modification de l'article 195, voy. notamment les contributions suivantes, classées en ordre chronologique: J. Masquelin, «La technique des révisions constitutionnelles en droit public belge », Annales de Droit et de Sciences Politiques, 1952, p. 89-129; A. Mast, « Het eerste bedrijf van de grondwetsherziening », Tijdschrift voor Bestuurswetenschappen en Publiekrecht, 1954, p. 59-72 ; A. Mast, «Une constitution du temps de Louis-Philippe », Revue du droit public, 1957, p. 987-1030 ; F. Delpérée, «La Constitution et la règle de droit », Annales de droit [de Louvain], 1972, p. 187-206 ; A. Vanwelkenhuyzen, «La procédure de révision de la Constitution et l'autorité des précédents en droit public belge », in : Miscellanea W.J. Ganshof van der Meersch, tome 3, Bruxelles, Bruylant, 1972, p. 329-350 ; A. Mast, «De grondwetsherziening naar Belgisch recht », in : De grondwet honderdvijftig jaar, Ontmoeting 7 februari 1981, Bruxelles, Bruylant, 1981, p. 129-145 ; H. Simonart, « La Révision de la Constitution », in : F. Delpérée (dir.), La Belgique fédérale, Bruxelles, Bruylant, 1994, p. 15-45 ; F. Delpérée, «La révision de la Constitution et la codification », in : L'Etat de Droit, Mélanges en l'honneur de Guy Braibant, Paris, Dalloz, 1996, p. 135-147. De plus amples références peuvent être trouvées dans la contribution mentionnée à la note 5 , supra.

${ }^{11}$ Même si le Constituant belge n'est, d'un point de vue strictement juridique, pas obligé de donner suite à la déclaration de révision du 9 avril.

${ }^{12}$ J.-Cl. Scholsem, « Brèves réflexions sur une éventuelle révision de l'article 195 de la Constitution », précité, p. 103.
} 
5. - Afin d'historiquement enraciner le débat (IV et V), nous ferons précéder celui-ci d'un bref aperçu tant de la genèse de l'article 195 (II) que des propositions les plus importantes qui ont été faites dans le passé en vue de sa modification (III).

\section{II - L'ARTICLE 195 : UN TEXTE PRESQUE BICENTENAIRE (1815-2003)}

6. - Les origines de l'article 195 de la Constitution belge peuvent être retracées jusqu'en 1815, année du vote de la Loi fondamentale des Pays-Bas réunis ${ }^{13}$ dont les articles 229 à 234 contiennent des dispositions similaires ${ }^{14}$. Celles-ci prévoient le vote d'une déclaration de révision ${ }^{15}$, une procédure bicamérale au niveau de la révision proprement dite ${ }^{16}$, l'intervention du Souverain ${ }^{17}$, un quorum de présences ${ }^{18}$ et un seuil particulier de majorité ${ }^{19}$. Malgré toutes ces similitudes, une différence notable existe : la Loi fondamentale de 1815 ne prévoit pas la dissolution automatique des Chambres après l'adoption d'une déclaration de révision ${ }^{20}$.

7. - Après l'Indépendance belge ${ }^{21}$, le Constituant de 1830-1831 - Constituant dont on sait par ailleurs qu'il s'est beaucoup inspiré de la Loi fondamentale des Pays-Bas réunis ${ }^{22}$ - n'a guère accordé à la question de la modification de la Constitution l'importance que celle-ci revêt pourtant naturellement dans toute démocratie constitutionnelle. Ainsi, quand Charles Raikem voulut présenter, le 24 janvier 1831, en tant que rapporteur, son exposé sur le problème en question, le Congrès national en ordonna simplement « l'impression et la distribution (...) sans vouloir en entendre la parole $»^{23}{ }^{24}$. Quelques jours plus tard, le 4 février, lors de la séance du vote définitif du Titre VII de la Constitution intitulé « De la Révision »- titre comprenant le seul article 131 - il en alla de même : la disposition fut adoptée «sans changement ${ }^{25}$ en un tour de main. Le Congrès avait plus important à faire ${ }^{26}$.

C'est ainsi que la règle de la dissolution automatique des Chambres suite au vote de la déclaration de révision - non contenue dans la Loi fondamentale néerlandaise et aujourd'hui située au centre des débats ${ }^{27}$ - fut intégrée dans l'article 131 sans le moindre commentaire : trouvant sa source dans le 'Projet de Constitution $^{28}$ élaboré par la Commission de Constitution $^{29}$ au mois d'octobre 1830 en cinq jours

\footnotetext{
${ }^{13}$ Du 24 août 1815 (Journal Officiel [du Royaume des Pays-Bas], 28 août, supplément au numéro 29).

${ }^{14}$ Voy. O. Orban, op.cit., tome 2, p. 703-704 et 709 où il évoque les «liens de filiation qui existent entre notre art. 131 et les art. 229 et suivants de la Loi fondamentale hollandaise du 24 août 1815 ». - Voy. aussi A. Verhasselt-Luykx, op.cit., p. 230.

${ }^{15}$ Voy. article 229

${ }^{16}$ Voy. articles 230 et 232, dernière phrase. L'article 78 de la Loi fondamentale prévoit en effet que les Etats généraux se composent de deux Chambres, à savoir de la Seconde Chambre (articles 79 et 81-86) et de la Première Chambre (articles 80 et 87-89).

${ }^{17}$ Voy. article 232, dernière phrase.

${ }^{18}$ Voy. article 232, première phrase. Il s'agit d'un quorum des deux tiers, mais seulement applicable à la Seconde Chambre.

${ }^{19}$ Voy. article 232, première phrase. Il s'agit du seuil des trois quarts des voix exprimées ; il semble cependant - même si cette question reste à approfondir - que celui-ci s'applique à la seule Seconde Chambre.

${ }^{20}$ Voy. O. Orban, op.cit., tome 2, p. 710, J. Masquelin, op.cit., p. 90-91, et A. Verhasselt-Luykx, op.cit., p. 230.

${ }^{21}$ Voy. la Proclamation de l'Indépendance par le Gouvernement provisoire, en date du 4 octobre 1830 (Bulletin officiel, n ${ }^{\circ} 4$ ), et le Décret constitutionnel du Congrès national du 18 novembre 1830 (Bulletin officiel, $\mathrm{n}^{\circ} 41$ ).

${ }^{22}$ Selon l'étude de Gilissen, quarante pour cent des dispositions de la Constitution belge de 1831 sont plus ou moins textuellement empruntées à la Loi fondamentale des Pays-Bas de 1815 (voy. J. Gilissen, «La Constitution belge de 1831 : ses sources, son influence », Res Publica, numéro spécial [hors série] intitulé Les problèmes constitutionnels de la Belgique au XIXe siècle, 1968, p. 107-141, ici 132).

${ }^{23}$ E. Huyttens, Discussions du Congrès national de Belgique, tome 2, Bruxelles, Société topographique belge, 1844, p. 252.

${ }^{24}$ Ce rapport peut être trouvé dans E. Huyttens, op.cit., tome 4, p. 110-112 (pièce $n^{\circ} 63$ ). - En définitive, l'appréciation de van Nieuwenhove selon laquelle « l'Assemblée plénière du Congrès National a accordé peu d'attention à la disposition qui règle la révision de la Constitution » est encore un euphémisme (J. van Nieuwenhove, op.cit., p. 20. C'est nous qui traduisons).

${ }^{25}$ E. Huyttens, op.cit., tome 2, p. 461.

${ }^{26}$ Notamment de se choisir un Roi, le Duc de Nemours ayant été choisi par le Congrès le 3 février, mais ayant fait connaître son refus le 17 du même mois (voy. séances des 3 et 21 février 1831, E. Huyttens, op.cit., tome 2, p. 455 et 550-552).

${ }^{27}$ Voy. infra, $\mathrm{n}^{\text {os }} 25-26$.

${ }^{28}$ E. Huyttens, op.cit., tome 4, p. 43-49 (pièce n ${ }^{\circ} 45$ ).
} 
seulement ${ }^{30}$, elle ne fut plus jamais rediscutée dans la suite. Ceci permet d'affirmer - aspect jusqu'ores guère souligné par la doctrine - que si tous les grands ouvrages de droit constitutionnel belge, à commencer par celui d'Oscar $\operatorname{Orban}^{31}$, contiennent classiquement trois justifications pour la règle de la dissolution automatique des Chambres ${ }^{32}$, il semble bien s'agir, à strictement parler, d'une rationalisation exclusivement menée a posteriori ${ }^{33}$.

8. - Le peu d'attention lors de son élaboration n'a pas empêché l'article 131 de jouir d'une longévité maximale. Deux Guerres mondiales, six Rois et presque autant de réformes de l'Etat ont été incapables d'en altérer le moindre iota : son libellé est toujours celui approuvé par le Congrès national. Et c'est ainsi que la Belgique vit ${ }^{34}$ encore à l'heure actuelle avec cette curieuse refonte des articles 229 à 234 de la Constitution néerlandaise de 1815 dont il constitue le produit fini.

\section{III - LES TENTATIVES DE MODIFICATION DE L'ARTICLE 195 (1892-2003)}

9. - L'impression d'inaltérabilité qui se dégage de la section précédente doit cependant être atténuée par un certain nombre de suggestions de modification de l'article 195 émises au cours du temps, tant d'origine parlementaire que doctrinale. S'il faut convenir avec Jean-Claude Scholsem que ces propositions «sont si nombreuses qu'on a peine à les suivre toutes ${ }^{35}$, il importe d'en indiquer malgré tout certaines ${ }^{36}$, particulièrement marquantes : le débat d'aujourd'hui (IV et V) ne peut en effet se mener sans avoir à l'esprit les précédents des 110 dernières années. Ceux-ci font dans un certain sens partie de la vie juridique de l'article 195.

10. - Les deux premières suggestions de modification - restées comme toutes les autres sans succès - sont d'origine parlementaire.

En février 1892, cinq députés déposent une proposition de déclaration de révision de cinq articles de la Constitution, dont l'article $131^{37}$. La révision de cette dernière disposition est demandée «afin de permettre à la constituante de stipuler que, à l'avenir, toute demande de révision devra comprendre l'indication $d u$ texte à mettre en lieu et place du texte à modifier $»^{38}$. Selon les auteurs, une telle modification de la procédure de révision permettrait d' « écarte[r] toute équivoque, en mettant la législature en présence d'un texte précis qu'elle pourra examiner en connaissance complète de cause. » ${ }^{39}$ Après

\footnotetext{
${ }^{29}$ Commission de quatorze membres, créée par Arrêté du Gouvernement provisoire du 6 octobre 1830 (voy. E. Huyttens, op.cit., tome 4, p. 42 [pièce $\left.\left.n^{\circ} 44\right]\right)$.

${ }^{30}$ Entre le 12 et le 16 octobre 1830. Au cours de cette période, la Commission de Constitution a tenu dix séances (voy. J. Gilissen, op.cit., p. 112113).

${ }^{31}$ O. Orban, op.cit. (en 3 tomes, rédigés respectivement en 1906, 1908 et 1911).

${ }^{32}$ Nous énumérerons ces trois justifications infra, $\mathrm{n}^{\circ} 25$.

33 Cette circonstance revêt également un intérêt pour le constitutionnaliste luxembourgeois, puisque le Constituant grand-ducal a presqu'intégralement recopié la Constitution belge. La seule différence entre l'article 131 de la Constitution belge et la disposition luxembourgeoise correspondante - l'article 114 de la Constitution du 17 octobre 1868 - se situe au niveau du quorum requis : tandis que la première exige la présence des deux tiers des membres, la seconde en requiert celle des trois quarts. Il échet par ailleurs de rappeler que le Grand-Duché ne dispose en raison de sa petite taille que d'un système parlementaire monocaméral.

${ }^{34}$ Tel est également le cas de nos voisins luxembourgeois, qui ont 'importé' l'article 131 de la Constitution belge (voy. supra, note précédente).

${ }^{35}$ J.-Cl. Scholsem, « Brèves réflexions sur une éventuelle révision de l'article 195 de la Constitution », précité, p. 103.

${ }^{36}$ Il s'agit donc bien d'une sélection, nullement exhaustive. - Nous signalons par ailleurs ne pas avoir pris en considération les propositions de modification de l'article 195 introduites par des partis extrémistes, visant à mettre fin à l'existence même de l'Etat belge. Ainsi, des propositions introduites au motif que « les organes constituants de la nation flamande souveraine devront régler l'entrée en vigueur et les révisions ultérieures de la Constitution » nous semblent se situer en dehors du débat (sur cette dernière proposition, voy. F. Delpérée, «Le processus de modification de la Constitution belge », précité, p. 75).

${ }^{37}$ Proposition de déclaration de révision des articles 6, 15, 23, 104 et 131 la Constitution, déposée à la séance plénière de la Chambre des Représentants le 16 février 1892 (Annales parlementaires, Chambre des Représentants, session ordinaire 1891-1892, p. 623, séance du 16 février 1892).

${ }^{38}$ Annales parlementaires, Chambre des Représentants, séance indiquée à la note précédente, p. 627 (C'est nous qui soulignons.).

${ }^{39}$ Ibidem (C'est nous qui soulignons.).
} 
l'intervention du Gouvernement ${ }^{40}$ la proposition fut cependant retirée ${ }^{41}$ et ne figure dès lors pas dans la déclaration de révision du mois de mai de la même année ${ }^{42}$.

11. - La seconde proposition est formulée en octobre 1919: cette fois-ci, c'est effectivement le préconstituant qui déclare l'article 131 ouvert à révision ${ }^{43}$. Si cet épisode retient toujours l'attention de la doctrine contemporaine ${ }^{44}$, c'est parce qu'il a constitué jusqu'en avril 2003 l'unique occasion où l'article 131 s'est trouvé ouvert à révision.

Donnant suite à des propositions doctrinales émises en ce sens ${ }^{45}$, le préconstituant de 1919 désirait clairement - comme le démontrent les travaux parlementaires ${ }^{46}$ - supprimer le mécanisme de la dissolution automatique des Assemblées et remplacer cette formalité par celle d'un référendum constitutionnel national sur la révision projetée ${ }^{47}$. Son intention n'était donc nullement d'atténuer la lourdeur de la procédure prescrite par l'article 131 .

12. - Les troisième et quatrième suggestions de modification de l'article 131 sont quant à elles d'origine doctrinale.

Dans les deux cas, le constat de base est le même : la procédure de révision prévue par l'article 131 s'applique à la révision de toute disposition constitutionnelle quelle qu'elle soit; il n'y a pas de révision possible en dehors des limites tracées par l'article 131. C'est en ce sens que la procédure revêt un caractère uniforme. Or, dans l'après-guerre, la doctrine propose à deux reprises - et pour des motifs différents l'abandon de cette uniformité, en adjoignant à la lourde procédure de l'article 131 une autre, plus souple, réservée à des cas particuliers.

La première suggestion trouve son origine dans une intervention de Walter Ganshof van der Meersch de 1953, dans laquelle il relève qu'une modification des articles 25 et 68 de la Constitution belge ${ }^{48}-$ modification permettant des transferts de souveraineté - serait susceptible de « détourner ${ }^{49}$ la procédure de l'article 131 elle-même. Il se demande dès lors s'il ne convient pas «d'inscrire également l'article 131 parmi les articles qui doivent faire l'objet d'une révision $»^{50}$. En filigrane se profile ainsi la question de savoir s'il ne faut pas créer une procédure moins lourde de révision de la Constitution spécifiquement pour le cas où la Belgique voudrait donner son assentiment à un traité international directement applicable qui serait, sans une révision préalable de la Constitution, incompatible avec celle-ci. Le débat s'est poursuivi jusqu'à l'heure actuelle ${ }^{51}$ et revêt certainement un intérêt pour la révision projetée en 2003. Nous y reviendrons $\left(\mathrm{n}^{\circ} 30\right)$.

\footnotetext{
${ }^{40}$ Voy. le discours de M. de Burlet, ministre de l'Intérieur et de l'Instruction publique, Annales parlementaires, Chambre des Représentants, session ordinaire 1891-1892, p. 712-717, séance du 25 février 1892.

${ }^{41}$ Déclaration du député Coremans, Annales parlementaires, Chambre des Représentants, session ordinaire 1891-1892, p. 718, séance du 25 février 1892).

${ }^{42}$ Déclaration de révision de la Constitution du 23 mai 1892 (Moniteur belge, 23-24 mai).

${ }^{43}$ Déclaration de révision de la Constitution du 22 octobre 1919 (Moniteur belge, 23 octobre).

${ }^{44}$ Voy. notamment J. van Nieuwenhove, op.cit., p. 51-52, 106 et 116, et J.-Cl. Scholsem, « Brèves réflexions sur une éventuelle révision de 1'article 195 de la Constitution », précité, p. 100 et note 4.

${ }^{45}$ Voy. O. Orban, op.cit., tome 2, p. $724-725$ et $727-728$.

${ }^{46}$ Voy. Documents parlementaires, Chambre des Représentants, session ordinaire 1918-1919, p. 1164-1169 (document $\mathrm{n}^{\circ} 329$ ) et p. 1580-1589 (document $\mathrm{n}^{\circ}$ 424), Documents parlementaires, Sénat, session ordinaire 1918-1919, p. 143-144 (document $\mathrm{n}^{\circ} 231$ ), Annales parlementaires, Chambre des Représentants, session ordinaire 1918-1919, p. 1947-1956, séance du 8 octobre 1919, et Annales parlementaires, Sénat, session ordinaire 1918-1919, p. 859-867, séance du 15 octobre 1919.

${ }^{47}$ Documents parlementaires, Chambre des Représentants, $\mathrm{n}^{\circ} 329$, précité, contenant l'exposé des motifs fait, au nom du Gouvernement, par le ministre de l'Intérieur de Broqueville, p. 1168. - Parallèlement, le gouvernement comptait introduire, au niveau des lois ordinaires, le mécanisme de référendum législatif (voy. Documents parlementaires, Chambre des Représentants, même document, précité).

${ }^{48}$ Il s'agit des articles 33 et 167 de la Constitution coordonnée.

${ }^{49}$ Actes de la Treizième Journée Interuniversitaire d'Etudes Juridiques, Annales de Droit et de Sciences Politiques [de Louvain], 1953, p. 299-330, ici p. 325.

${ }^{50}$ Ibidem (C'est nous qui soulignons.).

${ }^{51}$ Voy. notamment la récente intervention de F. Delpérée, «La déclaration de révision de la Constitution : le 195, certainement pas, un 195bis, pourquoi pas? », Journal des Procès, n 450 (10 janvier 2003), p. 16-17. - Voy. par ailleurs P. Brouwers et H. Simonart, « Le conflit entre la Constitution et le droit international conventionnel dans la jurisprudence de la Cour d'arbitrage », Cahiers de droit européen, 1995, p. 7-22, ici p.
} 
13. - L'autre suggestion d'une procédure parallèle à l'article 131 fut émise par Pierre Wigny. Employant des termes très vagues, cet auteur émet au début des années 1960 la thèse selon laquelle il conviendrait de distinguer parmi les dispositions de la Constitution belge trois catégories de normes : celles dotées d'une « valeur supraconstitutionnelle $»^{52}$, celles dotées d'une valeur $《$ infraconstitutionnelle ${ }^{53}$ et celles dotées d'une valeur constitutionnelle ${ }^{54}$. Les premières dispositions ne peuvent être supprimées - même pas par l'application de l'article $131^{55}$ - mais peuvent être révisées ${ }^{56}$. Les deuxièmes et troisièmes, quant à elles, peuvent être supprimées et révisées : conformément à l'article $131{ }^{57}$ pour les dispositions 'simplement' constitutionnelles et par une procédure allégée ${ }^{58}$ pour les dispositions infraconstitutionnelles.

Présentant une certaine affinité avec la Wesentlichkeitstheorie en droit constitutionnel allemand ${ }^{59}$, la théorie de Wigny ne résiste pas à certaines objections : d'une part, on est en droit de se demander si toute disposition constitutionnelle ne revêt pas - du moins sous certains aspects - un caractère essentiel par le fait même de se trouver dans la Loi fondamentale. D'autre part - et ceci constitue peut-être le contre-argument le plus important - la répartition des dispositions de la Constitution en différentes catégories - en l'absence d'une liste claire opérant cette distinction - requiert, pour être efficace, la création d'un organe chargé de la vérification de la catégorisation exacte des dispositions constitutionnelles dont la révision est projetée. Or, il n'est guère besoin de souligner que dans la Belgique des années 1960, époque où même le contrôle du Pouvoir législatif n'était pas encore organisé ${ }^{60}$, celui du Pouvoir constituant devait paraître passablement utopique.

Ne quittant jamais les hautes sphères d'un flou artistique évanescent, frôlant la contradiction ${ }^{61}$, la théorie de Wigny ne convainc guère, et l'on doit convenir avec Herman van Impe que « la logique interne de la théorie de Pierre Wigny laisse (...) à désirer ${ }^{62}$. Mais malgré toutes ses vicissitudes, elle a eu la vie remarquablement dure : encore au milieu des années 1980, soit plus de trente ans après les propos initiaux, elle circulait toujours, quoique dans une version un tant soit peu 'démystifiéee ${ }^{63}$.

14. - La cinquième grande suggestion de modification de l'article 131 est émise en 1985 par le Centre d'études de la réforme de l'Etat, organe pouvant être situé à mi-chemin entre les sphères académique et parlementaire. Celui-ci recommande le maintien tant du mécanisme de la déclaration de révision que de

22, ainsi que Y. Lejeune et P. Brouwers, « La Cour d'arbitrage face au contrôle de constitutionnalité des traités », note sous Cour d'arbitrage, arrêt 26/91, Journal des Tribunaux, 1992, p. 671-676, ici p. 676.

${ }^{52}$ P. Wigny, op.cit., p. 25.

${ }^{53}$ P. Wigny, op.cit., p. 27.

${ }^{54}$ La théorie de Wigny pèche par imprécision. Ainsi, elle ne précise jamais combien de catégories de normes elle comprend exactement. Il nous semble cependant que si l'on postule l'existence de normes supraconstitutionnelles (c'est-à-dire situées au-dessus de la Constitution) et infraconstitutionnelles (c'est-à-dire situées en-dessous de celle-ci), l'on doive admettre comme troisième catégorie de normes les normes constitutionnelles tout court, catégorie qui sert de point de départ ou, autrement dit, de catégorie de référence pour les deux autres rubriques (l'une se situant au-dessus de celle-ci, l'autre en-dessous de celle-ci).

55 « Il paraît juste de reconnaître que même en respectant toutes les procédures de l'article 131, on ne peut supprimer [les dispositions de valeur supraconstitutionnelle]. » (P. Wigny, op.cit., p. 25).

56 « Leur formulation et leur aménagement (...) peuvent être modifiés par la procédure de la révision » (P. Wigny, op.cit., p. 25-26).

${ }^{57}$ Du moins il convient de le penser au vu de ce que nous avons dit supra, note 54.

58 «Sont au contraire infraconstitutionnelles, les dispositions qui ne bénéficient pas de la stabilité garantie par l'article 131 》 (P. Wigny, op.cit., p. 27 ; c'est nous qui soulignons.).

${ }^{59}$ Sur cette théorie, voy. J. Isensee et P. Kirchhof (éds.), Handbuch des Staatsrechts der Bundesrepublik Deutschland, tome 3, Heidelberg, C.F. Müller, 1988, p. 337-342, P. Badura, Staatsrecht - Systematische Erläuterung des Grundgesetzes für die Bundesrepublik Deutschland, Munich, C.H. Beck, 1986, p. 75-76 et p. 364-365, et E. Stein et G. Frank, Staatsrecht, $17^{\mathrm{e}}$ éd., Tübingen, Mohr Siebeck, 2000 , p. $153-154$.

${ }^{60}$ La Cour d'arbitrage de Belgique - juridiction constitutionnelle à compétence partielle - ne fut créée qu'en 1983.

${ }^{61} \mathrm{La}$ meilleure illustration de cette apparence de contradiction est la circonstance qu'après avoir affirmé, à propos des dispositions supraconstitutionnelles, que «leur formulation et leur aménagement (...) peuvent être modifiés par la procédure de la révision » (op.cit., 25-26), l'auteur énonce avec une imperturbable nonchalance que «[s]ont supraconstitutionnelles, les dispositions qui ne peuvent être soumises à la procédure de la révision» (op.cit., p. 27 ; c’est nous qui soulignons.). Le lecteur reste perplexe.

${ }^{62}$ H. van Impe, « De onlenigheid van de Belgische grondwet», Tijdschrift voor Bestuurswetenschappen en Publiekrecht, 1968, p. 18-21, ici p. 19. C'est nous qui traduisons.

${ }^{63}$ Voy. la proposition de J. Gol, mentionnée par F. Delpérée, « Une crise en trois temps », précité, p. 121-122. 
«la division du travail entre deux législatures $»^{64}$, mais se prononce en faveur de la suppression de la dissolution automatique des Chambres, une fois la déclaration de révision adoptée. Selon le Centre, cette suppression pourra éventuellement être compensée par l'instauration d'un «délai de réflexion d'un an minimum entre la déclaration de révision et la révision proprement dite. ${ }^{65}$

15. - L'alternance entre impulsions doctrinales et politiques est confirmée par la sixième proposition, à nouveau doctrinale. Si celle-ci ne vise pas directement l'article 131, elle mérite néanmoins d'être relevée. Après la réforme de l'Etat de 1993, Francis Delpérée suggère dans une contribution parue au cours de la même année de rendre irrévisable ${ }^{66}$ le nouvel article $1^{\mathrm{er}}$ de la Constitution, disposition qui stipule que « [1]a Belgique est un Etat fédéral qui se compose des communautés et des régions ${ }^{67}$. Si elle n'était pas restée lettre morte, cette proposition aurait eu une incidence directe sur l'article 131, puisqu'elle en aurait restreint le champ d'application ${ }^{68}$.

16. - Enfin, la septième et dernière grande suggestion de modification de l'article 195 est d'origine parlementaire et présente un lien étroit avec celle de 1919. En novembre 1995, les députés Dewael, Daems et Lano proposent d'ouvrir l'article 195 de la Constitution à révision ${ }^{69}$, afin «d'institutionnaliser le référendum non seulement pour les matières législatives, mais également pour les matières constitutionnelles $\gg^{70}$. Une déclaration de révision ayant cependant été votée quelques mois plus tôt seulement ${ }^{71}$, la proposition fut privée de toute chance de succès et ne fut pas non plus reprise dans la déclaration de révision suivante adoptée en mai $1999^{72}$.

17. - La filiation, la genèse (II) et le curriculum vitae (III) de l'article 195 étant ainsi établis, nous pouvons aborder la question de son libellé futur. Il nous semble qu'une réflexion sur son éventuelle formulation (V) doive partir de certaines prémisses (IV), tant générales (A) que spécifiques (B).

\section{IV - LES PREMISSES GENERALES ET SPECIFIQUES D'UNE NOUVELLE VERSION DE L'ARTICLE 195 - OU : COMMENT LA 'BOITE DE PANDORE’ SE VIDE PEU A PEU}

\section{A - PREMISSES GENERALES}

18. - Un tour de droit constitutionnel comparé suffit pour s'en convaincre, la procédure de modification de la Constitution doit de manière tout à fait générale satisfaire à trois préceptes: elle doit être plus contraignante que toute autre procédure législative de l'ordre juridique en question, son application doit rester réaliste ${ }^{73}$ et le mécanisme prévu - pour rigide qu'il soit - doit demeurer simple et clair.

\footnotetext{
64 «Synthèse des travaux que le Centre d'études de la réforme de l'Etat a consacrés à la procédure de révision de la Constitution », Document interne du Centre (en date du 24 juillet 1985), p. 3.

${ }^{65}$ Ibidem.

${ }^{66}$ « 'La forme fédérale de l'Etat n'est pas sujette à révision', pourrait-on écrire demain dans la Constitution » (F. Delpérée, « La Belgique est un Etat fédéral », Journal des Tribunaux, 1993, p. 637-646, ici p. 645).

${ }^{67}$ Modification à la Constitution du 5 mai 1993 (Moniteur belge, 8 mai).

${ }^{68} \mathrm{Si}$ bien que la modification de la Constitution suggérée par Francis Delpérée aurait préalablement nécessité l'ouverture à révision tant de l'article $1^{\mathrm{er}}$ que de l'article 195 de la Constitution. - Pour une réaction à la proposition de Delpérée, voy. M.-F. Rigaux, « Sur l'opportunité d'interdire de modifier la structure fédérale de 1'Etat par une clause constitutionnelle irréformable », Administration publique trimestrielle, 1994 , p. $243-246$.

${ }^{69}$ Documents parlementaires, Chambre des Représentants, session ordinaire 1995-1996, n 276/1 (document en date du 29 novembre 1995).

${ }^{70}$ Idem, p. 1 .

${ }^{71}$ Déclaration de révision de la Constitution du 12 avril 1995 (Moniteur belge, 12 avril).

${ }^{72}$ Déclaration de révision de la Constitution du 4 mai 1999 (Moniteur belge, 5 mai).

${ }^{73}$ Nous entendons par là une susceptibilité de mise en œuvre plus grande qu'une simple applicabilité théorique. - Pour un contre-exemple particulièrement marquant où un texte constitutionnel avait prévu une procédure de révision manifestement irréalisable en pratique, voy. le Titre XIII de la Constitution du 5 Fructidor de l'An III (22 août 1795), dont la mise en œuvre aurait dans le meilleur des cas nécessité plus de dix ans.
} 


\section{1 - La procédure de révision doit demeurer plus contraignante que les procédures de modification de normes législatives}

19. - Il découle de la première prémisse que, sous peine de bouleverser la structure hiérarchisée de l'ordre juridique belge, la Constitution fédérale doit demeurer plus difficilement modifiable que les normes législatives, y compris celles adoptées à une majorité spéciale ${ }^{74}$. Mettre en cause cette exigence reviendrait à contester de facto la suprématie de la Constitution ${ }^{75}$, concept qui revêt pourtant une importance essentielle $^{76}$. Il s'ensuit que si les conditions exigées pour le vote d'une loi spéciale peuvent servir de base de départ pour une réflexion au niveau constitutionnel ${ }^{77}$, elles sont clairement insuffisantes pour la procédure de révision de la Constitution. Nous reviendrons ultérieurement sur ce point ( $\left.{ }^{\circ} 35\right)$.

\section{2 - La procédure de révision doit demeurer praticable}

20. - Etant l'expression d'un certain 'réalisme juridique', la seconde prémisse traite de la 'praticabilité' de la procédure de révision. Afin de se conformer à celle-ci, il importe notamment de ne pas exiger pour un amendement de la Constitution un seuil de majorité déraisonnablement élevé : s'il est pertinent de protéger la minorité contre une tyrannie de la majorite $e^{78}$, il en va incontestablement de même en sens inverse ${ }^{79}$. A cet égard, il faut signaler le risque de voir un seuil de majorité particulièrement draconien rendre des petits partis extrémistes numériquement indispensables à toute entreprise de révision constitutionnelle et d'exposer le Constituant à leur merci. Or, il faut à tout prix éviter que des forces antidémocratiques parviennent à museler le Constituant après avoir échoué à dominer le législateur ${ }^{80}$.

\section{3 - Le libellé de la procédure de révision doit demeurer clair et concis}

21. - La troisième prémisse ne nous semble guère nécessiter d'explication : l'exigence de rigidité doit aller de pair avec celles de clarté et de concision. La procédure d'amendement de la Constitution doit ainsi tenir en trois ou quatre phrases. Des formulations malencontreuses comme celle du nouvel article 151 inséré en 1998 - disposition et qui compte pas moins de 33 phrases, dont certaines contiennent de longues énumérations - sont à proscrire.

\section{$B$ - PREMISSES SPECIFIQUES}

22. - A ces trois prémisses générales, jouissant d'une validité abstraite - indépendante de l'ordre juridique en question - semblent s'ajouter trois autres, spécifiques, tirées des particularismes de l'ordre juridique belge.

\footnotetext{
${ }^{74}$ Une loi adoptée à la majorité spéciale (appelée 'loi spéciale') est une norme législative fédérale adoptée en respectant les conditions de majorité et de présences stipulées au dernier alinéa de l'article 4 de la Constitution coordonnée, c'est-à-dire «adoptée à la majorité des suffrages dans chaque groupe linguistique [néerlandais et français] de chacune des Chambres [Chambre des Représentants et Sénat], à la condition que la majorité des membres de chaque groupe [linguistique] se trouve réunie et pour autant que le total des votes positifs émis dans les deux groupes linguistiques atteigne les deux tiers des suffrages exprimés. »

${ }^{75}$ Voy. F. Delpérée, Le Droit constitutionnel de la Belgique, Bruxelles et Paris, Bruylant et L.G.D.J., 2000, p. 62 : « La loi spéciale ne peut être assimilée à la Constitution. (...) Elle ne saurait avoir valeur constitutionnelle ; en particulier, il ne lui appartient pas de modifier sans habilitation l'ordre des attributions constitutionnelles » (C'est l'auteur qui souligne.). Cette position est d'autant plus justifiée que toute loi belge, même votée à la majorité spéciale, peut faire l'objet d'un contrôle de constitutionnalité devant la Cour d'arbitrage. Voy. en ce sens Cour d'arbitrage, arrêts $8 / 90$ (considérants B.2.2. et B.2.4.), 17/94 (considérant B.5.3., in fine), 24/98 (considérants B.9.2. et svts.) et 118/98 (considérants 3 et 4 ).

${ }^{76}$ Voy. J. Limbach [Présidente de la Cour constitutionnelle allemande], « The concept of the Supremacy of the Constitution », Modern Law Review, vol. 64, 2001, p. 1-10, et F. Delpérée, Le Droit constitutionnel de la Belgique, précité, p. 87-95.

${ }^{77}$ En ce sens, M. Uyttendaele, Précis de Droit constitutionnel belge, précité, p. 108.

${ }^{78}$ Voy. notamment C. Daubie, «Les techniques de protection des minorités », Annales de droit [de Louvain], 1972, p. 207-244, spécialement le chapitre intitulé 'La protection des minorités et la révision constitutionnelle', p. 217-243.

${ }^{79}$ Sur le problème de la discrimination de la majorité, par des mesures visant à assurer la non-discrimination d'une minorité, voy. J. Rubenfeld, « The Anti-Antidiscrimination Agenda », Yale Law Journal, vol. 111, 2002, p. 1141-1178.

${ }^{80}$ En Belgique, la pertinence de cette prémisse générale et abstraite est fort bien démontrée par la situation au sein du collège électoral néerlandais (où le parti flamand d'extrême droite occupe 16,5 pc des sièges néerlandophones à la Chambre des Représentants, soit 15 députés néerlandophones sur 91).
} 


\section{1 - La nouvelle procédure de révision ne contiendra pas de techniques référendaires}

23. - La première semble être l'impossibilité politique, dans la Belgique de nos jours, d'insérer dans la procédure de révision de la Constitution une quelconque forme de référendum. Si Xavier Delgrange et Hugues Dumont font état de leur préférence pour une telle solution ${ }^{81}$, nous estimons à l'instar de A. Verhasselt-Luykx ${ }^{82}$, André Alen ${ }^{83}$, Jean-Claude Scholsem ${ }^{84}$, et Marc Uyttendaele ${ }^{85}$ que le référendum national est politiquement inconcevable en raison tant de la répartition inégale de la population en termes d'appartenance linguistique ${ }^{86}$ que de motifs historiques indéniables ${ }^{87}$.

\section{2 - La nouvelle procédure de révision continuera à exclure les entités fédérées}

24. - La seconde prémisse spécifique nous semble consister en ce que la compétence normative ${ }^{88}$ de révision de la Constitution demeure exclusivement fédérale. Cette solution s'impose pour deux raisons, l'une théorique, l'autre pratique.

La raison théorique consiste en ce que la participation des entités fédérées au processus de révision de la Constitution n'est pas une conséquence nécessaire du caractère fédéral d'un Etat. Ainsi, la République fédérale d'Allemagne et la République d'Autriche - Etats dont le caractère fédéral est incontesté - ne permettent pas à leurs entités de prendre part, en tant que telles, à la modification de la Constitution fédérale $^{89} 90$. Cette solution est par ailleurs parfaitement cohérente, puisque dans ces Etats, les entités

\footnotetext{
${ }^{81}$ Ceci non sans audace, circonstance que les auteurs semblent bien admettre eux-mêmes, puisqu'ils considèrent leurs présuppositions « sans doute largement 'contre-factuelles' » (voy. X. Delgrange et H. Dumont, op.cit., p. 468). Hugues Dumont avait déjà antérieurement fait état de sa position favorable par rapport au mécanisme du référendum constitutionnel (voy. ses contributions «Etat, Nation et Constitution. De la théorie du droit public aux conditions de viabilité de l'Etat belge », Belgitude et crise de l'Etat belge, Actes du colloque organisé par la Faculté de droit des Facultés universitaires Saint-Louis le 24 novembre 1988, Bruxelles, Publications des Facultés universitaires Saint-Louis, 1989, p. 73-124, spécialement p. 105-107, et « La réforme de 1993 et la question du référendum constituant », Administration publique trimestrielle, 1994, p. 101-107).

${ }^{82}$ Cette liste est établie de manière chronologique. - A propos d'une éventuelle instauration d'un référendum constituant, A. Verhasselt-Luykx constate déjà en 1971 que «l'existence des deux communautés linguistiques semble constituer un obstacle infranchissable pour le bon déroulement d'une procédure objective de référendum » (A. Verhasselt-Luykx, op.cit., p. 238. C'est nous qui traduisons.) .

83 Ainsi, André Alen note que « des référendums au niveau fédéral, portant par exemple sur des problèmes linguistiques ou communautaires, semblent impossibles dans la Belgique bipartite» (A. Alen, op.cit., p. 110 ; c'est nous qui traduisons. Nous insistons sur le mot « impossible ».).

${ }^{84}$ J.-Cl. Scholsem, « Brèves réflexions sur une éventuelle révision de l'article 195 de la Constitution », précité, p. 104.

${ }^{85}$ M. Uyttendaele, Précis de Droit constitutionnel belge, précité, p. 109-111, et, déjà antérieurement, «Le référendum constitutionnel en Belgique ou une réponse inadaptée à une question pertinente », Administration publique trimestrielle, 1994, p. 109-114, spécialement p. 112-114.

${ }^{86}$ Avec, sur les 10.296 .350 personnes habitant le Royaume au $1^{\text {er }}$ octobre 2001, 5.968.074 domiciliées en région de langue néerlandaise (soit 57,96 $\%$ ), 3.283.407 en région de langue française (soit 31,89\%), 973.565 en région bilingue de Bruxelles-Capitale (soit 9,46 \%), et 71.304 en région de langue allemande (soit 0,69\%). Source : Moniteur belge, 28 mai 2002 ( 2 édition).

${ }^{87}$ A cet égard, la consultation populaire du 12 mars 1950 relative à la «Question Royale » nous semble constituer une contre-indication dirimante par rapport à toute tenue de référendum national en Belgique. Allant dans le même sens, Marc Uyttendaele considère que l'«[o]n ne peut évoquer la question du référendum en Belgique sans se référer à la loi du 11 février 1950 qui institua une consultation populaire au sujet de la question royale » (M. Uyttendaele, «Le référendum constitutionnel en Belgique ou une réponse inadaptée à une question pertinente », précité, p. 114, note 17). Dans le même sens, voy. A. Alen, Belgisch Staatsrecht, précité, p. 110.

88 Nous entendons par 'compétence normative de la révision de la Constitution' la compétence de poser des actes indispensables à l'accomplissement de la procédure de révision. La question de savoir quelles entités devraient être admises à participer à l'exercice de la compétence normative de révision est distincte de celle de savoir quelles entités devraient être dotées d'une compétence non normative en la matière (telle une compétence d'avis ou de consultation), problème dont nous ne traitons pas. Par ailleurs, il faut noter que si une simple compétence d'avis revêt en principe un caractère non normatif, il en irait cependant autrement dans l'hypothèse où la procédure de révision stipulerait, sans fixer de délai, que telle ou telle entité doit nécessairement émettre un avis et qu'aucune révision ne pourra avoir lieu sans l'accomplissement de cette formalité.

${ }^{89} \mathrm{Il}$ importe de souligner que tant le Bundesrat allemand que le Bundesrat autrichien sont des organes exclusivement fédéraux : ce n'est pas parce que tant en Allemagne qu'en Autriche, la Constitution fédérale attribue aux différents Länder un quote-part fixe du nombre total des parlementaires de cette Seconde Chambre (il s'agit là de dispositions qui ont pour seul but de régler le mode de recrutement des parlementaires), que le Bundesrat deviendrait un organe fédéré. En effet, le fonctionnement et le statut des parlementaires de la Seconde Chambre - avec tout leur régime de droit de vote, d'immunités et de privilèges - sont exclusivement régis par la Constitution et la législation fédérales, si bien que ses parlementaires sont, tout comme leurs collègues de la Première Chambre fédérale, des parlementaires fédéraux et non des parlementaires fédérés (cette dernière appellation devant être réservée aux mandataires siégeant dans les différents Landtag, c'est-à-dire les parlements des Länder). Il apparaît ainsi que pour pouvoir parler d'une participation directe des entités fédérées au processus de révision constitutionnelle, la proposition de révision projetée devrait être soumise à un vote dans chaque parlement fédéré, c'est-à-dire dans chacun des seize Landtag des Länder de la République fédérale ou des neuf Landtag des Länder de la République d'Autriche. Or, les parlements des Länder allemands et autrichiens ne sont pas autorisés à se prononcer sur une telle proposition, la révision de la Constitution étant adoptée par le seul Pouvoir législatif fédéral (le Bundestag et le Bundesrat en Allemagne et le Nationalrat et le Bundesrat en Autriche), ce qui constitue bien la preuve que les entités fédérées allemandes et autrichiennes ne participent pas directement au processus de la révision de la Constitution.

${ }^{90}$ Voy. l'article 79, alinéa 2, de la Loi fondamentale allemande du 23 mai 1949 et les articles 10 , alinéa $1^{\text {er }}$, $1^{\circ}$, et 44 , alinéas $1^{\text {er }}$ et 2 , de la Loi fédérale constitutionnelle autrichienne du 10 novembre 1920.
} 
fédérées disposent elles-mêmes de Constitutions fédérées. Au vu de ce dernier aspect, l'affirmation de Karel Rimanque selon laquelle en Belgique «l'implication des composantes de la fédération [dans le processus de révision constitutionnelle] demeure insuffisante pour un Etat qui dans l'article premier de sa Constitution s'annonce comme 'Etat fédéral' $\gg{ }^{91}$ ne semble pas s'imposer: si quelque chose est «insuffisant » pour les entités fédérées ${ }^{92}$, c'est n'est pas leur non-participation à la modification de la Constitution fédérale mais le degré de leur autonomie constitutive fédérée ${ }^{93}$.

La raison pratique de conserver le caractère exclusivement fédéral de la compétence de révision peut être énoncée comme suit. Dans l'état actuel des choses, la Belgique compte sept entités distinctes ${ }^{94}$, chacune dotée de compétences exclusives. Conférer à toutes une compétence normative propre dans le processus de révision de la Constitution (fédérale) - et partant un véritable droit de veto ${ }^{95}$ - rendrait la procédure impraticablement lourde ${ }^{96}$. Or le problème du nombre des entités fédérées ne peut être résolu par une limitation à certaines entités seulement: quelle que soit l'ingéniosité de sa conception, une telle idée se brise systématiquement sur des arguments juridiques dirimants ${ }^{97}$.

Le maintien de la compétence de révision entre les mains du seul Etat fédéral se révèle donc non seulement conceptuellement justifié mais aussi pratiquement indiqué. Cette solution n'empêchera par ailleurs ni l'instauration, dans le nouvel article 195, d'une exigence d'une majorité dans chaque groupe linguistique ${ }^{98}$, ni une influence indirecte des entités fédérées, par exemple au travers d'un Sénat réformé ${ }^{99}$.

\section{3 - La nouvelle procédure de révision sera moins lourde que celle de l'actuel article 195}

25. - Venons-en à la dernière prémisse spécifique. Si l'article 195 de la Constitution belge est depuis fort longtemps déjà exposé à une critique sans cesse croissante et aujourd'hui presque généralisée, c'est avant tout en raison du fait que l'on estime de plus en plus que la procédure de révision prescrite par cette disposition revêt un caractère excessivement lourd. Or, il est intéressant de se demander par quel élément procédural précis, au sein du libellé de l'article 195, cet alourdissement aujourd'hui jugé insupportable est principalement généré. Formulé de manière quelque peu plus imagée, on peut ainsi s'interroger où se cache, sur le vieux navire de la révision de la Constitution belge, la 'boule de plomb' qui le fait couler.

\footnotetext{
${ }^{91}$ K. Rimanque, De grondwet toegelicht, gewikt en gewogen, Anvers, Intersentia, 1999, p. 382. C'est nous qui traduisons.

${ }^{92}$ Supposition sur le bien-fondé de laquelle nous ne pouvons nous prononcer dans le cadre du présent exposé.

${ }^{93}$ Autonomie qui dans l'état actuel du droit semble trop étroite pour rendre possible la naissance de Constitutions fédérées. - Pour de plus amples développements de ce point, voy. J.-Cl. Scholsem, "Faut-il une Constitution pour chaque entité fédérée? Réflexions sur le phénomène constitutionnel dans un Etat fédéral » (1995), disponible en version électronique à l'adresse www.wallonie-en-ligne.net/wallonie-politique/1995cife_wallonie-region_europe/CIFE14.htm, C. Berx, « De ruime grondwetgevende bevoegdheid van deelstaten : een rechtsvergelijkende studie », Anvers, Maklu, 1994, 208 p., J. Clement, W. Pas, B. Seutin, G. van Haegendoren et J. van Nieuwenhove, Proeve van Grondwet voor Vlaanderen, Bruges, Die keure, 1996, 353 p., et C. Mertes, «L'autonomie constitutive des Communautés et des Régions », Courrier hebdomadaire du CRISP, $\mathrm{n}^{\circ} 1650-1651,1999,60 \mathrm{p}$.

${ }^{94}$ L'Etat fédéral, les Communautés flamande, française et germanophone, la Région wallonne, la Région de Bruxelles-Capitale et la Commission communautaire commune. Dans ce calcul, il est déjà fait abstraction de la Commission communautaire française, alors que celle-ci est dotée - du moins partiellement - d'un pouvoir décrétal.

${ }^{95}$ Voy. supra, note 88.

${ }^{96}$ Circonstance déjà indirectement soulignée par M. Uyttendaele, Précis de Droit constitutionnel belge, précité, p. 110.

${ }^{97}$ D'un point de vue théorique, la limitation des entités fédérées autorisées à participer au processus de révision de la Constitution pourrait s'opérer de trois manières différentes: on pourrait restreindre la participation au processus de révision aux seules grandes entités [c'est-à-dire aux Communautés flamande et française, ainsi qu'à la Région wallonne](a), aux seules Communautés (b) ou encore aux seules Régions (c). Chacune de ces trois formules se heurte cependant à des objections juridiques insurmontables. Ainsi, le modèle (a) est irréaliste car l'absence d'une fusion entre la Communauté française et la Région wallonne - alors qu'une telle fusion est intervenue entre les Communauté et Région flamandes - créerait une disproportion manifeste dans les rapports numériques Nord-Sud, une seule entité fédérée néerlandophone étant appelée à contribuer à la modification de la Constitution, alors que la partie francophone du pays le serait au travers de deux entités. Quant à la solution (b), elle est démographiquement inacceptable étant donné qu'elle conférerait un droit de veto à la minuscule Communauté germanophone qui ne représente pourtant que 0,69 \% de la population du Royaume. Enfin, la formule (c) est politiquement 'invotable' en raison tant de la non-existence de la Région flamande comme entité fédérée distincte que du fait que la classe politique néerlandophone est très attachée à l'idée que la Région de Bruxelles-Capitale présente certaines spécificités, lui conférant un statut différent, quelque peu subalterne, par rapport aux autres Régions du pays.

${ }^{98}$ Exigence que l'actuel article 195 ne contient pas. Une adaptation de la procédure de révision aux réalités linguistiques du pays nous semble cependant nécessaire (en ce sens aussi M. Uyttendaele, Précis de Droit constitutionnel belge, précité, p. 108 et 112). - Voy. aussi infra, $\mathrm{n}^{\circ} 36.2$.

${ }^{99}$ Sur la réforme projetée du Sénat, voy. notamment La Libre Belgique, version informatisée (www.lalibre.be), 19 et 22 avril 2002.
} 
Or, il n'est guère contesté que c'est précisément la partie la plus récente, c'est-à-dire le seul morceau véritablement belge ${ }^{100}$ de l'article 195, qui y apporte le surpoids aujourd'hui fatal : la dissolution automatique des Chambres après le vote de la déclaration de révision. Il apparaît ainsi qu'un allègement efficace de la procédure de révision nécessite une ré-évaluation sévère - et c'est un euphémisme - de la règle.

Depuis longtemps, la formalité de la dissolution automatique attire les critiques d'une très large partie de la doctrine constitutionnelle belge. Ainsi Jean-Claude Scholsem souligne que «l'adoption de la déclaration de révision entraîne la dissolution automatique des Chambres [constitue] la difficulté principale ${ }^{101}$ de la procédure de révision, Xavier Delgrange et Hugues Dumont affirment que la dissolution automatique des Chambres constitue « la règle la plus critiquée tant pour sa lourdeur que pour son inefficacité » ${ }^{102}$, Marc Uyttendaele constate que la procédure de révision «n'est plus guère adaptée » et se demande « s'il ne convient pas de [la] revoir ${ }^{103}$, et Philippe Brouwers et Henri Simonart réclament ouvertement « [1]'abrogation pure et simple de la règle qui veut que l'adoption d'une déclaration entraîne la dissolution automatique des Chambres fédérales ${ }^{104}$. La liste pourrait encore être allongée ${ }^{105}$.

Cette désapprobation doctrinale se fonde à la fois sur des raisons pratiques et théoriques. Sur le plan pratique, il faut faire état de nombreuses tentatives de «contournement ${ }^{106}$, privant de tout sens le mécanisme de la dissolution des Chambres. Sur le plan théorique, il faut admettre que deux ${ }^{107}$ des trois justifications traditionnellement invoquées à son appui « relèvent (...) aujourd'hui de la pure fiction ${ }^{108}$ et que même la troisième ${ }^{109}$ ne possède plus qu'une «validité limitée » ${ }^{110}$.

Mais ce qu'il importe avant tout de souligner, c'est la circonstance que le mécanisme est non seulement critiqué à l'heure actuelle, mais qu'il est exposé à une contestation durable depuis presque cent ans déjà : comme l'a démontré le bref aperçu de la vie juridique de l'article 195 (III), l'opportunité de la dissolution automatique des Chambres est mise en question dès $1908^{111}$, puis en $1919,1968^{112}, 1985,1995$ et à l'heure actuelle, en $2003^{113}$. Compte tenu à la fois de cette imposante chaîne d'éléments historiques et de la contestation actuelle dont le mécanisme de la dissolution automatique des Chambres fait l'objet, une révision de l'article 195 sans toucher à la règle instaurant la dissolution de plein droit des Chambres paraît inconcevable.

\footnotetext{
${ }^{100}$ Voy. supra, $\mathrm{n}^{\text {os }}$ 6-7.

${ }^{101}$ J.-Cl. Scholsem, « Brèves réflexions sur une éventuelle révision de l'article 195 de la Constitution », précité, p. 100.

${ }^{102}$ X. Delgrange et H. Dumont, op.cit., p. 454.

${ }^{103}$ M. Uyttendaele, Précis de Droit constitutionnel belge, précité, p. 108. - Dans une contribution antérieure, cet auteur écrivait par ailleurs que « [1] dissolution de plein droit des chambres n'implique pas forcément - c'est un euphémisme - un débat institutionnel devant les électeurs et les résultats du scrutin ne peuvent éclairer les représentants de la Nation sur la manière d'orienter le processus de révision » (M. Uyttendaele, « Le référendum constitutionnel en Belgique ou une réponse inadaptée à une question pertinente », précité, p. 111).

${ }^{104}$ P. Brouwers et H. Simonart, o.c., p. 22.

${ }^{105}$ Voy. notamment Y. Lejeune et P. Brouwers, o.c., p. 676. - Voy. cependant contra (comme seule voix discordante, semble-t-il) C. Carette, « La problématique de la 'rigidité' de la Constitution », La Revue politique, 1992, n 2, p. 37-51, ici p. 46) qui affirme qu' « aucun argument convaincant ne semble pouvoir être dégagé pour initier une modification de l'article 131 en vue de l'introduction d'une plus grande souplesse ».

${ }^{106}$ M. Uyttendaele, Précis de Droit constitutionnel belge, précité, p. 106. - Pour un aperçu détaillé du problème, voy. X. Delgrange et H. Dumont, op.cit., p. 437-453. - A cet égard, il convient notamment de mentionner le caractère très vague des déclarations de révision, intervenant par ailleurs à l'extrême fin de la législature (sur ce dernier point, voy. aussi J.-Cl. Scholsem, «Brèves réflexions sur une éventuelle révision de l'article 195 de la Constitution », précité, p. 101, n 3 ).

${ }^{107}$ Ces deux justifications étant d'une part, de rendre les parlementaires très attentifs à l'importance d'une révision de la Constitution et d'autre part, de permettre au peuple de donner, dans une certaine manière, son assentiment au projet de révision via les élections (voy. J.-Cl. Scholsem, «Brèves réflexions sur une éventuelle révision de l'article 195 de la Constitution », précité, p. 100-101).

${ }^{108}$ J.-Cl. Scholsem, « Brèves réflexions sur une éventuelle révision de l'article 195 de la Constitution », précité,p. 101.

${ }^{109}$ A savoir le souci de «protéger la Constitution, œuvre stable par définition, des emballements d'une majorité peut-être massive, mais éphémère. Il en va de l'essence même de toute Constitution, garante du droit des minorités » (J.-Cl. Scholsem, op.cit., 101).

${ }^{110}$ J.-Cl. Scholsem, « Brèves réflexions sur une éventuelle révision de l'article 195 de la Constitution », précité, p. 101.

111 Voy. supra, note 45.

${ }^{112}$ Pour des raisons de place, nous n'avons pas examiné cette date dans la partie consacrée aux tentatives de modification de l'article 131. Nous nous permettons de renvoyer le lecteur à la contribution indiquée supra, note 5.

${ }^{113}$ Voy. notamment le $\mathrm{n}^{\circ} 16$ de la contribution de H. Dumont, X. Delgrange et S. van Drooghenbroeck «La procédure de révision de la Constitution : suggestions », La procédure de révision de la Constitution [belge], Actes du colloque organisé par la Faculté de droit de l’Université catholique de Louvain le 14 février 2003, Bruxelles, Bruylant (à paraître).
} 
26. - Notre troisième prémisse spécifique consiste dès lors en un allègement de la procédure de révision, se traduisant par la suppression de l'exigence de la dissolution de plein droit des Chambres.

27. - En synthèse, nous estimons que l'éventuelle formulation nouvelle de l'article 195 devra prendre en considération les éléments suivants : le maintien d'une rigidité plus élevée que celle requise pour la modification de toute autre norme de l'ordre juridique belge, le rejet de formules impraticables, la clarté et la concision textuelle de la procédure, l'exclusion de toute forme de référendum du processus de révision, le maintien de la compétence exclusivement fédérale en la matière - en tenant toutefois compte de l'existence de groupes linguistiques au sein des Chambres fédérales - et l'allègement de la lourdeur de la procédure de révision, par la suppression de la dissolution automatique des Chambres.

Au vu de ce qui précède, un constat s'impose : la boîte de pandore s'est largement vidée.

\section{V - ENTRE RIGIDITE EXCESSIVE ET SOUPLESSE AUTODESTRUCTRICE : UNE INCURSION DANS LE 'LEBENSRAUM JURIDIQUE' DU FUTUR ARTICLE 195}

28. - C'est à partir de ces six points-balises qu'il convient d'examiner le problème du libellé futur de l'article 195, problème à propos duquel Xavier Delgrange et Hugues Dumont soulignent que « si la doctrine est quasi unanime pour (...) dénoncer les défauts [de l'actuelle formulation de l'article 195], elle ne montre guère d'empressement ni de conviction à en proposer une meilleure ${ }^{114}$.

La retenue doctrinale est cependant justifiée. Comme l'a souligné H.W.R. Wade de Cambridge, le constitutionnaliste remplit au mieux sa fonction s'il «avoids attempting to give legal answers to political questions ${ }^{115}$. Allant dans le même sens, Francis Delpérée note qu' «il ne revient pas aux spécialistes de la science constitutionnelle d'anticiper sur les choix en opportunité politique ${ }^{116}$. Partant, nous ne pouvons nous fixer comme objectif de présenter une formulation toute faite du nouvel article 195 : cette tâche doit être réservée aux trois branches du Pouvoir constituant.

Ce que le constitutionnaliste peut cependant faire - et ce qui fera l'objet de la présente section - est de se livrer à une réflexion encadrante sur les possibilités qui s'offrent au Constituant belge. A cet égard, la question centrale est de savoir de quelle marge de manœuvre celui-ci dispose réellement au vu des six indications prémisses susmentionnées.

\section{A - FAUT-IL MAINTENIR LE CARACTERE UNIFORME DE REVISION?}

29. - Le premier point que le Constituant sera appelé à trancher concerne le maintien ou non du caractère uniforme de la procédure de révision de la Constitution, c'est-à-dire la question de savoir s'il ne convient pas, dans des cas précis, de mettre en place une procédure plus souple de révision. Si cette problématique est susceptible de revêtir un intérêt dans plusieurs domaines juridiques ${ }^{117}$, nous nous limiterons dans le cadre de la présente étude à l'examen d'un seul de ceux-ci, à savoir au droit communautaire.

30. - L'hypothèse précise dans laquelle l'instauration d'un mécanisme différencié de révision peut être envisagée dans le domaine de la construction européenne est celle où l'assentiment belge à une modification des traités fondateurs se heurterait au niveau interne à l'exigence d'une modification préalable

\footnotetext{
${ }^{114}$ X. Delgrange et H. Dumont, op.cit., p. 453.

${ }^{115}$ H.W.R. Wade, « The Basis of Legal Sovereignty », Cambridge Law Journal, 1955, p. 197 (C'est nous qui soulignons.).

${ }^{116}$ F. Delpérée, « Avant-propos : Quelle déclaration de révision de la Constitution? », Revue belge de Droit constitutionnel, 1999, p. 4.

${ }^{117}$ Ce point est examiné plus en détail dans la contribution indiquée supra, à la note 5.
} 
de la Constitution. La question se pose de savoir si dans ce cas il ne convient pas de mettre en place une procédure plus souple de révision. Trois observations - une juridique, une historique et une socioéconomique - peuvent être faites à cet égard.

D'un point de vue juridique, il faut souligner la difficulté particulière des mécanismes différenciés de révision, difficulté qui réside en l'identification et la délimitation des normes auxquelles la procédure simplifiée est applicable. La théorie de Pierre Wigny ${ }^{118}$ en fournit une illustration marquante : visant à instaurer une procédure simplifiée de révision pour les seules normes infraconstitutionnelles, elle omettait de procéder à une détermination précise de cette catégorie de normes. Or, un tel problème est insusceptible de se poser dans le contexte de la construction européenne : les normes en question - les traités fondateurs - sont très aisément identifiables ${ }^{119}$.

Le second aspect est historique. Depuis 1831 - et essentiellement depuis 1945 - la configuration politique européenne a été bouleversée au point que l'on peut parler d'un véritable 'change out of recognition,'120. Dès lors, la circonstance que le Constituant de 1831 ait opté pour une procédure uniforme de révision ne constitue guère une indication utile pour trancher la question de savoir si aujourd'hui, 170 ans plus tard et dans un monde où tout a changé, il convient de faire de même ${ }^{121}$.

Enfin, il serait également simplificateur de mener la discussion sans faire allusion à un élément, il est vrai purement factuel mais néanmoins fondamental, à savoir le contexte socio-économique dans lequel elle se meut. Si en 1831 il était encore possible de considérer l'Etat belge comme entité souveraine d'un point de vue économique, financier et budgétaire, la construction européenne a fait fondre la Belgique dans un ensemble plus grand, impliquant l'effacement des frontières douanières et monétaires ainsi que - élément capital - la perte de la souveraineté budgétaire. C'est précisément parce que les obligations européennes assez astreignantes ${ }^{122}$ semblent entièrement compensées par les avantages que le pays en tire ${ }^{123}$ que l'on peut se demander s'il n'irait pas du propre intérêt de la Belgique d'instaurer des mécanismes permettant une adaptation plus souple du droit constitutionnel interne à d'éventuelles modifications des traités fondateurs. De tels mécanismes existent en Autriche ${ }^{124}$ et en Finlande ${ }^{125}$, et la Constitution belge - à l'instar de la Constitution française - contient elle-même des dispositions spécifiquement consacrées à l'intégration européenne ${ }^{126}$.

31. - Nous avons tenté de très brièvement le démontrer, une installation de mécanismes différenciés de révision peut à certaines conditions s'avérer pertinente. Si certains auteurs se sont exprimés en faveur de la

\footnotetext{
${ }^{118}$ Voy. supra, $\mathrm{n}^{\circ} 13$.

${ }^{119}$ Il existe à l'heure actuelle (mai 2003) trois traités fondateurs, à savoir le traité CE de 1957, le traité CEEA de la même année et le traité sur l’Union européenne de 1992. - Le traité CECA de 1951 a expiré le 23 juillet 2002.

${ }^{120}$ A cet égard, il faut avant tout mentionner le concept de supranationalité, apparu au cours de la seconde moitié du XX ${ }^{\mathrm{e}}$ siècle. - Sur cette notion, voy. notamment K. von Lindeiner-Wildau, La supranationalité en tant que principe de droit, Leiden, Sijthoff, 1970 , 175 pages, et C. Amirante, Unioni sovranazionali e riorganizzazione constituzionale dello stato, Turin, Giappichelli, 2001, 162 pages. En droit belge, voy. M. Melchior, « Constitution et organisations internationales. Le problème de la supranationalité », Le nouveau droit constitutionnel, Rapports belges au II ${ }^{e}$ Congrès mondial de droit constitutionnel, Louvain-la-Neuve et Bruxelles, Academia et Bruylant, 1987, p. 322-339.

${ }^{121}$ A cet égard, un élément essentiel du débat tient en la création, en 1983, d'une juridiction constitutionnelle, capable de censurer les lois. Les situations antérieures et postérieures à cette date diffèrent en effet fondamentalement : alors qu'avant la mise en place de la Cour d'arbitrage, le législateur, doté d'impunité totale, était de facto en mesure de déroger aux normes constitutionnelles sans avoir à procéder à leur révision formelle, il est depuis 1983 obligé juridiquement - et non seulement politiquement - au respect de la celles-ci. Cette altération dans les rapports entre Constitution et législateur fait également apparaître sous un nouveau jour la procédure de révision de la Constitution : là où il suffisait naguère de simplement adopter une loi, il peut être nécessaire aujourd'hui de formellement réviser la Constitution.

${ }^{122}$ A cet égard, l'on pense avant tout aux quatre critères de convergence stipulés au paragraphe $1^{\text {er }}$ de l'article 121 (ex-article 109 J) du traité instituant la Communauté européenne (Journal officiel des Communautés européennes, C 191 du 29 juillet 1992).

${ }^{123}$ Ainsi, la balance commerciale belge des échanges intracommunautaires démontre que le pays vit véritablement de l'intégration européenne (voy. Office national [belge] de statistique, tableau « Commerce extérieur et balance commerciale [1997-2001] », disponible en version électronique à l'adresse http://statbel.fgov.be/figures/d72_fr.asp ).

${ }^{124}$ Voy. les articles 9, alinéa 2, et 50, alinéa 3, de la Loi fédérale constitutionnelle autrichienne du 10 novembre 1920.

${ }^{125}$ Voy. le $§ 94$, alinéa 2, et le $§ 95$, alinéa 2, de la nouvelle Loi fondamentale finlandaise du 11 juin 1999 (entrée en vigueur le $1^{\text {er }}$ mars 2000 ).

${ }^{126}$ Il s'agit de l'article 168 de la Constitution coordonnée, inséré en 1993 (Moniteur belge, 8 mai). - Par contre, les articles 34 et 169 de la Constitution coordonnée ne sont, quant à eux, pas limités au seul droit communautaire mais ont un champ d'application plus large (l'article 34 fut inséré en 1970 [Moniteur belge, 18 août], l'article 169 en 1993 [Moniteur belge, 8 mai]).
} 
préservation d'une procédure uniforme ${ }^{127}$ - rejetant par là des procédures différenciées - nous pensons que des positions en sens inverse ${ }^{128}$ seraient tout aussi défendables. C'est en cela - ni plus, ni moins - que consiste notre conclusion de la présente section.

\section{B - FAUT-IL MAINTENIR LA FORMALITE DE LA DECLARATION DE REVISION?}

32. - La seconde question que le Constituant sera appelé à trancher touche à la technique de la déclaration de révision : dès lors que l'on postule l'abrogation du mécanisme de la dissolution automatique des Chambres ${ }^{129}$, se justifie-t-il encore de conserver la formalité de la déclaration de révision ? Il convient de présenter les différentes solutions concevables.

\section{1 - Conservation de la formalité de la déclaration de révision}

33. - Examinons en premier lieu l'hypothèse du maintien de la formalité de la déclaration de révision, tout en procédant - par application de la troisième prémisse spécifique - à la suppression du mécanisme de la dissolution automatique des Chambres. A cet égard, deux sous-hypothèses sont à distinguer.

33.1. - La première consiste en un scénario conservateur à l'excès : la seule modification apportée à la procédure de révision serait la suppression pure et simple - c'est-à-dire sans remplacement par une nouvelle exigence procédurale - des alinéas 2 et 3 de l'article 195 qui instaurent l'obligation de la dissolution automatique des Chambres ${ }^{130}$. Il est cependant évident qu'un tel modèle ne pourra être retenu : celui-ci permettrait au Pouvoir constituant de voter une déclaration de révision aujourd'hui, de la faire publier au Moniteur belge demain et d'amender la Loi fondamentale le surlendemain. La réforme préjudicierait ainsi gravement à l'indispensable rigidité - et partant à la stabilité - de la Constitution ${ }^{131}$.

33.2. - Contrairement à la première sous-hypothèse, le second modèle ne se borne pas à l'abolition pure et simple de la dissolution automatique des Chambres mais prévoit des mécanismes destinés à contrebalancer celle-ci. Un exemple concret peut être trouvé dans les travaux du Centre pour la réforme de l'Etat de 1985 132: la suppression de la dissolution automatique des Chambres y est compensée par l'exigence d'un étalement obligatoire du processus de révision sur deux législatures ${ }^{133}$. Préservant la rigidité de la Constitution, la formulation envisagée en 1985 nous semble toujours parfaitement envisageable aujourd' hui 134.

34. - Les deux modèles précédents se placent, pour reprendre l'expression de Jean-Claude Scholsem, dans une « optique minimaliste de [la] révision de l'article $195{ }^{135}$ : la formalité de la déclaration de révision y est en effet maintenue. Or, il faut bien avouer qu'une telle option ne s'impose pas nécessairement. A l'instar du mécanisme de la dissolution automatique des Chambres, la technique de la déclaration de révision a elle aussi fait l'objet de nombreuses critiques ${ }^{136}$. Dirigées avant tout contre le caractère souvent trop vague des déclarations, elles peuvent être retracées jusqu'en $1892^{137}$.

\footnotetext{
${ }^{127}$ En ce sens, Y. Lejeune et P. Brouwers, op. cit., p. 676, et P. Brouwers et H. Simonart, op.cit., p. 22.

${ }^{128}$ Pour ce qui est du droit européen, voy. en ce sens l'intervention de F. Delpérée, mentionnée supra, à la note 51.

${ }^{129}$ Ceci conformément à la troisième prémisse spécifique (voy. supra, $\mathrm{n}^{\text {os }} 25-26$ ).

${ }^{130}$ Le texte de l'article 195 figure supra, au n ${ }^{\circ} 2$.

${ }^{131}$ A cet égard, même l'instauration d'une exigence de majorité dans chaque groupe linguistique - en plus de celle d'une majorité des deux tiers des voix exprimées au total - nous semble insuffisante, étant donné que la seule dissimilitude entre la révision de la Constitution, conçue dans ces termes, d'une part et une modification de la loi spéciale d'autre part consisterait en un vote supplémentaire, à la majorité ordinaire, et précédant peut-être juste de quelques jours - le vote principal. Or, une telle procédure de révision ne protège qu'insuffisamment la rigidité de la Constitution, pourtant essentielle.

${ }^{132}$ Voy. supra, $\mathrm{n}^{\circ}$ 14, et J.-Cl. Scholsem, « Brèves réflexions sur une éventuelle révision de l'article 195 de la Constitution », précité, p. 102.

${ }^{133}$ La déclaration de révision et la révision ne pouvant être adoptées au cours de la même législature, sans que la déclaration de révision entraîne pour autant la dissolution automatique des Chambres.

${ }^{134}$ Pour autant qu'elle soit complétée - ajout cependant très aisé à réaliser - par l'exigence d'une majorité dans chaque groupe linguistique (voy. supra, $\left.\mathrm{n}^{\circ} 24\right)$.

135 J.-Cl. Scholsem, « Brèves réflexions sur une éventuelle révision de l'article 195 de la Constitution », précité,p. 103.

${ }^{136}$ Ainsi Marc Uyttendaele souligne que «l'énumération limitative des articles sujets à révision conduit à des incohérences qui se traduisent, par exemple, par des modifications implicites de la Constitution et par la coexistence, au sein de celle-ci, de dispositions contradictoires » (M.
} 


\section{2 - Suppression de la formalité de la déclaration de révision}

35. - Il convient par conséquent d'examiner la marge de manœuvre du Constituant dans l'hypothèse d'un abandon tant de la dissolution automatique que de la déclaration de révision. A cet égard, il importe de signaler qu'une suppression simultanée pure et simple des deux mécanismes précités, sans instaurer des mesures compensatoires, est inconcevable : elle mènerait tout simplement à la formule applicable aux lois spéciales ${ }^{138}$.

Mais dès lors qu'il convient d'eviter que la Constitution ne devienne aussi facilement modifiable que la loi spéciale $^{139}$, des mesures compensatoires, susceptibles de maintenir une difficulté supplémentaire d'amendement au niveau de la Constitution doivent être trouvées. Celles-ci peuvent être de deux natures : elles peuvent se situer soit au niveau de la majorité requise $\left(\mathrm{n}^{\circ} 36\right)$, soit au niveau de la procédure applicable $\left(\mathrm{n}^{\circ} 37\right)$.

36. - Dans le domaine des conditions de majorité, la recherche d'une difficulté d'amendement supplémentaire pourrait s'opérer à deux niveaux, à savoir au seuil de la majorité globale $\left(\mathrm{n}^{\circ} 36.1\right)$ et au pourcentage de la majorité dans chaque groupe linguistique $\left(\mathrm{n}^{\circ} 36.2\right)^{140}$.

36.1 - Quant aux conditions applicables à la majorité globale, un certain nombre d'éléments déjà mentionnés plus haut ${ }^{141}$ semble indiquer qu'il ne convient pas d'excéder le seuil des deux tiers, ceci en vue d'éviter que le concours de partis extrémistes ne devienne indispensable l'entreprise de révision constitutionnelle.

La nécessité de ne pas excéder le seuil des deux tiers ne priverait cependant pas le Constituant de toute marge de manœuvre : s'inspirant des articles 46 et 96 de la Constitution, on pourrait imaginer de démarquer la procédure de révision de la procédure de modification de la loi spéciale non pas au niveau du seuil de la majorité requise, mais au niveau de la base de départ pour le calcul de celle-ci. Ainsi, les majorités requises pour l'amendement de la Constitution pourraient être calquées non pas sur la base des voix exprimées donnée par essence variable - mais sur celle, stable et légèrement plus sévère, des membres ${ }^{142}$.

Deux éléments nous semblent plaider en faveur d'une telle suggestion. D'une part, il convient d'admettre avec Paul Errera qu' «il va de soi que, pour un événement aussi grave qu'un changement constitutionnel, on pourra compter sur la présence de la presque totalité des députés et des sénateurs ${ }^{143}$. D'autre part, le critère des membres présenterait le considérable avantage de rendre inutile toute exigence de quorum ${ }^{144}$, circonstance qui ferait non seulement renforcer la concision et la clarté textuelle de l'article 195 mais qui

Uyttendaele, Précis de Droit constitutionnel belge, précité, p. 108), et Xavier Delgrange et Hugues Dumont notent que « [1]a règle de la déclaration préalable contraint les Chambres à faire preuve d'une préscience dont elles sont nécessairement dépourvues. En pratique, dans les conditions politiques qui sont celles d'aujourd'hui, pour identifier judicieusement tous les articles dont l'ouverture à révision se révélera souhaitable, elles devraient deviner les termes de l'accord politique requis pour atteindre la majorité des deux tiers nécessaire pour faire œuvre constituante, alors que cet accord ne pourra être conclu qu'après les élections sous la législature suivante par des partenaires qu'elles ne sauraient identifier puisque leur sélection est en partie tributaire de ces élections qui n'ont, par hypothèse, pas encore eu lieu » (X. Delgrange et H. Dumont, op.cit., p. 427-428).

${ }^{137}$ Voy. supra, $\mathrm{n}^{\circ} 10$.

${ }^{138}$ Pourvu que l'on instaure l'exigence d'une majorité dans chaque groupe linguistique, ce que nous présumons conformément à la seconde prémisse spécifique (voy. supra, $\mathrm{n}^{\circ} 24$ ).

${ }^{139}$ Ceci conformément à la première prémisse (énoncée supra, ${ }^{\text {os }} 18-19$ ), qui stipule que la Constitution doit demeurer la norme la plus difficilement modifiable de l'ordre juridique belge. Nous y insistons.

${ }^{140}$ Il faut cependant souligner l'interdépendance des deux seuils.

${ }^{141}$ Voy. supra, $\mathrm{n}^{\circ} 20$.

${ }^{142}$ Le seuil de majorité requise pour l'amendement de la Constitution serait ainsi fixé à deux tiers des membres dans chaque chambre, et à la majorité des membres dans chaque groupe linguistique, par opposition à la loi spéciale dont la modification exige la majorité deux tiers des voix exprimées dans chaque chambre et à la majorité des voix exprimées dans chaque groupe linguistique (voy. aussi supra, note 74).

${ }^{143}$ P. Errera, Traité de Droit public belge, 2 édition, Paris, Giard \& Brière, 1918, p. 21-22.

${ }^{144}$ L'actuelle version de l'article 195 exige pour une révision de la Constitution un quorum des deux tiers des membres de chaque Chambre. L'article 4 de la Constitution qui fixe, quant à lui, le quorum pour une modification d'une loi spéciale, requiert la présence de la majorité des membres de chaque groupe linguistique. 
rendrait également sans objet la longue controverse doctrinale - entre-temps close ${ }^{145}$ - relative à la prise en compte ou non des abstentions : si l'on calque les exigences de majorité sur la base des membres, il est en effet incontestable qu'une majorité globale des deux tiers exige 100 votes positifs à la Chambre ${ }^{146}$ et 48 au Sénat ${ }^{147}$, et qu'une majorité dans chacun des groupes linguistiques se traduit par un assentiment de respectivement 30 francophones ${ }^{148}$ et 46 néerlandophones ${ }^{149}$ à la Chambre et de 15 francophones ${ }^{150}$ et 21 néerlandophones ${ }^{151}$ au Sénat. Le mécanisme excellerait par sa simplicité.

36.2 - Quant aux conditions de majorité dans chaque groupe linguistique, il convient avant toute chose de rappeler qu'il s'agit là d'exigences que l'actuel article 195 ne contient pas : datant d'un temps où toute la vie politique belge ne se faisait en qu'une seule langue - le français - l'actuelle procédure de révision de la Constitution est 'linguistiquement agnostique' ou, pour utiliser le langage des constitutionnalistes américains, 'language blind'. Si nous discutons malgré tout de la question de savoir quel seuil de majorité devrait être d'application aux groupes linguistiques, c'est parce que nous présumons conformément à la seconde prémisse spécifique $\left(\mathrm{n}^{\circ} 24\right)$ que la modification de la procédure de révision procédera à une adaptation de l'article 195 aux réalités politiques du pays, par l'installation de conditions de majorité applicables à chaque groupe linguistique.

Si la tâche de la détermination précise de ces exigences de majorité doit être réservée au monde politique, il échet cependant de relever que le danger relatif à l'influence potentielle des partis extrémistes - danger déjà exposé au niveau de la majorité globale $\left(\mathrm{n}^{\circ} 36.1\right)$ - vaut encore a fortiori au niveau des groupes linguistiques, puisque la présence de partis anti-démocratiques accuse un important déséquilibre communautaire : alors que l'on ne compte, au sein du groupe linguistique français à la Chambre des Représentants, qu'un député extrémiste sur 59, on en dénombre 15 sur 91 (soit plus de 16 pour cent) au sein du groupe néerlandais. Au vu de ces chiffres, toute suggestion visant à instaurer, au niveau des groupes linguistiques, des exigences de majorité plus lourdes que celle de la majorité ordinaire doit être précédée d'un soigneux exercice de calcul, faute de quoi l'oeuvre du Constituant - garant suprême de la démocratie et des libertés - risquerait d'être exposée au bon vouloir des forces anti-démocratiques. C'est pour cette raison que nous estimons devoir situer le maximum maximorum du seuil de majorité applicable à chaque groupe linguistique séparément à soixante pour cent, soit à trois cinquièmes ${ }^{152}$.

36.3 - Les modifications qui viennent d'être suggérées ( $\mathrm{n}^{\text {os }} 36.1$ et 36.2) sont toutefois à elles seules insuffisantes pour garantir l'indispensable rigidité de la procédure de révision : il convient par conséquent de rechercher des pistes relevant du domaine strictement procédural, afin d'assurer une temporisation, un espacement dans le temps du processus de révision.

\footnotetext{
${ }^{145}$ Pour un bon résumé de celle-ci, voy. A. Vanwelkenhuyzen, «La procédure de révision de la Constitution et l'autorité des précédents en droit public belge », précité, et X, «Le calcul des majorités dans les assemblées délibérantes : le problème des abstentions », Courrier hebdomadaire du CRISP, $\mathrm{n}^{\circ} 424,1968,23 \mathrm{p}$.

${ }^{146}$ La Chambre des Représentants compte 150 membres au total. La répartition de ses membres en groupes linguistiques étant susceptible de légèrement fluctuer après chaque élection législative, il est supposé pour le présent calcul que 91 de ses membres fassent partie du groupe linguistique néerlandais et que 59 appartiennent au groupe linguistique français.

${ }^{147}$ Le Sénat compte 71 membres, abstraction faite des sénateurs de droit. Contrairement au mécanisme applicable à la Chambre des Représentants, la répartition des sénateurs en groupes linguistiques est invariable : 41 relèvent du groupe linguistique néerlandais, 29 du groupe linguistique français et un membre élu (celui désigné par le Conseil de la Communauté germanophone) ne fait partie d'aucun groupe linguistique. - Ajoutons que les sénateurs de droit, membres non élus du Sénat, ne relèvent pas non plus d'un groupe linguistique, mais qu'ils s'abstiennent généralement de prendre part au vote. Pour cette dernière raison, il s'indique de faire systématiquement abstraction de ceux-ci pour nos calculs.

${ }^{148}$ Sur 59 députés francophones au total (chiffre datant de la $50^{\mathrm{e}}$ législature).

${ }^{149}$ Sur 91 députés néerlandophones au total (chiffre datant de la $50^{\mathrm{e}}$ législature).

${ }^{150}$ Sur 29 sénateurs francophones au total.

${ }^{151}$ Sur 41 sénateurs néerlandophones au total.

${ }^{152}$ Tout en sachant que déjà une telle exigence peut se situer à la limite de la praticabilité. Une majorité des trois cinquièmes au sein de chaque groupe linguistique signifie en effet que sur les 76 députés néerlandophones 'démocratiques', 55 devront soutenir la proposition de révision : un refus par seulement 22 d'entre eux - soit d'à peine 29 pour cent des députés flamands démocrates et de seulement 15 pour cent du nombre total des députés belges (nous insistons) - serait déjà fatal. Par ailleurs, il n'est guère besoin de souligner que le nombre des 76 mandataires démocratiques flamands à la Chambre des Représentants est susceptible d'être altéré par les prochaines élections législatives prévues pour le printemps 2003.
} 
37. - D'un point de vue procédural, une constellation doit particulièrement être évitée au niveau de la Constitution : c'est que la majorité - même celle des deux tiers au total et, le cas échéant, des trois cinquièmes dans chaque groupe linguistique - dispose du pouvoir de faire «tout, tout de suite ${ }^{153}$. Car s'il est déjà critiquable de voir des lois spéciales hâtivement concoctées au terme de négociations nocturnes et précipitamment votées sans débat parlementaire approprié ${ }^{154}$, il en va a fortiori ainsi au niveau constitutionnel : le danger serait trop grand de voir en l'espace de quelques jours seulement toute la structure de l'Etat belge fondamentalement et irrévocablement ${ }^{155}$ modifiée.

Or, un étalement dans le temps du processus de révision constitutionnelle serait impossible si la procédure de la révision se limitait à un seul vote des Chambres ${ }^{156}$ : l'idée même d'un espacement dans le temps présuppose l'existence de deux échéances, échéances qui pourront être distanciées l'une par rapport à l'autre par la fixation d'un certain délai ou d'une certaine condition procédurale. En définitive, la suppression simultanée tant du mécanisme de la dissolution automatique que de la déclaration de révision débouche, si l'on entend maintenir une difficulté d'amendement supplémentaire au niveau de la Constitution, sur l'absolue nécessité d'instaurer l'exigence d'un double vote ${ }^{157}$.

37.1 - Les contours précis d'un tel mécanisme de double vote demeurent sujets à discussion : on pourrait notamment imaginer l'instauration d'une obligation d'étaler les deux scrutins sur deux législatures proposition déjà émise dans les années quatre-vingt par le Centre pour la Réforme de l'Etat ${ }^{158}-$ ou encore envisager la fixation précise d'un délai minimum qui devra s'écouler entre les deux votes, sans qu'il soit cependant besoin de les étaler sur deux législatures différentes ${ }^{159}$. Si la première hypothèse est plus contraignante que la seconde, elle peut - assez paradoxalement - se révéler plus rapide, du moins dans certains cas.

37.2 - Quant à la tenue proprement dite du double scrutin, c'est-à-dire quant à procédure de vote applicable à chacun de ceux-ci, il existe pareillement deux possibilités : soit les deux scrutins sont soumis à des conditions de majorité exactement identiques, soit ils obéissent à des exigences de majorité différentes ${ }^{160}$.

Il est vrai que la seconde hypothèse (prévoyant des conditions de majorité différentes) revêt un certain attrait académique : elle permettrait d'imaginer un modèle de vote où serait exigée, lors du premier scrutin, la seule majorité dans chaque groupe linguistique - représentant en quelque sorte un assentiment distinct de chacun des deux 'volksgroepen' qui peuplent la Belgique - et, lors du second, la seule majorité des deux tiers au total - constituant en quelque sorte un vote de la Fédération entière. Mais malgré la beauté de ces constructions théoriques, il faut, hélas, se rendre à l'évidence qu'elle sera évincée par la première hypothèse, qui l'emportera, même s'il est exact que celle-là débouche sur un assez morne second tour de vote, purement répétitif et vidé de signification propre.

\footnotetext{
${ }^{153}$ Voy. J.-Cl. Scholsem, « Brèves réflexions sur une éventuelle révision de l'article 195 de la Constitution », précité, p. 102.

${ }^{154}$ A cet égard, nous pensons avant tout aux négociations communautaires qui ont précédé le vote des deux lois spéciales du 13 juillet 2001 (Moniteur belge, 3 août), commentées notamment par F. Delpérée, « Belgique. La nouvelle vague fédéraliste », cette revue, 2001 , p. 675-686.

${ }^{155}$ Il faut en effet relever que surtout le transfert de compétences du fédéral vers les entités fédérées constitue un processus de facto irréversible, puisqu'une fois une matière 'défédéralisée', sa réattribution au pouvoir fédéral devient une impossibilité politique, aussi excellentes que soient les raisons pour ce faire. Cette circonstance fut déjà relevée il y a onze ans par J.-Cl. Scholsem, "La réforme de l'Etat : une mise en perspective », Actualités du Droit, Revue de la Faculté de Droit de Liège, 1991, p. 267-287, ici p. 275.

${ }^{156}$ A cet égard, il faut souligner que l'approbation d'un texte constitutionnel par la Chambre et par le Sénat ne constitue pas un double vote : chaque assemblée ne votant qu'une seule fois, il s'agit d'un vote simple. Prétendre le contraire serait confondre double vote et bicaméralisme. Par ailleurs, si l'exigence d'un étalement dans le temps devait seulement s'appliquer à la période entre le vote à la Chambre et celui au Sénat (ou vice versa), la procédure de la révision de la Constitution ne se distinguerait que de manière insignifiante de la procédure de la modification de la loi spéciale, ce qui serait inadmissible (voy. supra, $\mathrm{n}^{\circ} 19$ ).

${ }^{157}$ C'est-à-dire d'un double vote au sein de chaque Chambre, ces deux votes étant espacés dans le temps. - Nous signalons à cet égard qu'un mécanisme de double vote nécessite dès le premier scrutin l'existence d'un texte précis de la nouvelle disposition constitutionnelle et que toute modification de son libellé après la tenue du premier vote sera impossible.

${ }^{158}$ Proposition déjà émise par le Centre pour la Réforme de l'Etat (voy. supra, ${ }^{\text {os }} 14$ et 33.2).

${ }^{159}$ Ce délai pourrait par exemple être fixé à douze mois, laps de temps qui correspond à celui proposé par le Centre pour la réforme de l'Etat en 1985 (voy. supra, $\mathrm{n}^{\circ}$ 14). - Nous signalons par ailleurs que le Constituant pourrait estimer nécessaire d'instaurer en outre un délai maximum dans lequel le double vote devra intervenir.

${ }^{160}$ Etant entendu que le Constituant devra trancher la question de savoir si les majorités prémentionées se calculent sur base des voix exprimées ou des membres.
} 
38. - En résumé, il revient au Pouvoir constituant belge de procéder à des arbitrages sur quatre points principaux : la préservation d'une procédure uniforme de révision, la conservation de la technique de la déclaration de révision, la détermination précise de la base ${ }^{161}$ des majorités, et enfin, l'installation de mécanismes de temporisation. Le Constituant pourrait par ailleurs envisager d'aligner la procédure bilingue $^{162}$ de la révision sur le caractère trilingue ${ }^{163}$ de la Constitution.

39. - Comme le démontre le grand nombre de considérations spécifiques du présent exposé considérations tirées de l'Histoire nationale ${ }^{164}$, de la structure institutionnelle ${ }^{165}$, de la composition démographique ${ }^{166}$ et de l'intégration supranationale du Royaume ${ }^{167}$ - le débat sur le futur libellé de l'article 195 de la Constitution demeure essentiellement une question 'belgo-belge'. S'il est vrai que la question de la détermination précise de la procédure de révision de la Constitution se pose dans tout Etat doté d'une Constitution codifiée, des éléments du droit comparé - aussi bénéfiques que ceux-ci puissent être dans de nombreux autres domaines - ne se révèlent guère concluants : un examen des procédures de révision applicables dans les autres pays de l'Union européenne ${ }^{168}$ et aux Etats-Unis révèle que celles-ci contiennent dans de nombreux cas soit des techniques référendaires ${ }^{169}$, soit des mécanismes prévoyant une dissolution automatique des Chambres ${ }^{170}$, soit des exigences plus légères que celles applicables en Belgique à la modification de la loi spéciale ${ }^{171}$, soit enfin des techniques particulières, basées sur l'Histoire nationale respective et non transposables en Belgique en raison de cette spécificité ${ }^{172}$. Les Etats-Unis ne pouvant pas non plus servir d'exemple ${ }^{173}$, il semble que les seuls pays dont le Constituant belge pourrait éventuellement s'inspirer soient la Suède - quoiqu'en partie seulement ${ }^{174}$ - et la Finlande ${ }^{175}$.

\footnotetext{
${ }^{161}$ Mais non du seuil. Publiekrecht, 1997, p. 297-307.

${ }^{163}$ Art. 189 de la Constitution, tel que modifié le 23 octobre 1991 (Moniteur belge, 31 octobre).

${ }^{164}$ Voy. supra, $\mathrm{n}^{\text {os }} 6-8$ et 23 , et note 87.

${ }^{165}$ Voy. supra, ${ }^{\text {os }} 19$ et 24, spécialement note 97.

${ }^{166}$ Voy. supra, note 86.

${ }^{167}$ Voy. supra, $\mathrm{n}^{\mathrm{0}} 30$.
}

${ }^{162}$ Il n'existe en effet pas de version en langue allemande de la déclaration de révision. - Sur ce point, voy. aussi H. Simonart, o.c., p. $27-29$ (n ${ }^{\text {os }} 30-$ 32), et plus généralement B. Bergmans, Le statut juridique de la langue allemande en Belgique, Louvain-la-Neuve, Cabay, 1986, p. 7, ainsi que J. van Nieuwenhove, « De minimis non curat praetor? Over de Duitse vertaling van normatieve teksten », Tijdschrift voor Bestuurswetenschappen en

${ }^{168}$ Quatorze pays dont il convient d'emblée d'écarter le Royaume-Uni et le Luxembourg. En effet, au Royaume-Uni, la distinction formelle entre norme constitutionnelle et norme législative fait défaut, de sorte qu'il n'existe pas de procédure de révision constitutionnelle proprement dite (sur ce sujet, voy. C. Behrendt, «La notion de 'parliamentary sovereignty' en droit constitutionnel anglais: fondements, paradoxes et problèmes contemporains », Revue de droit international et de droit comparé, 2002, 221-289). Le Luxembourg doit quant lui être écarté en raison du fait que l'article 114 de la Constitution grand-ducale de 1868 est basé sur l'article 131 de la Constitution belge de 1831 et qu'il contient à un minime détail près exactement le même libellé que celui-ci (voy. aussi supra, notes 33-34).

${ }^{169}$ Ainsi, en Irlande, toute révision constitutionnelle doit être approuvée par référendum (art. 46, alinéa 2, de la Constitution du $1^{\text {er }}$ juillet 1937). Un référendum constitutionnel dans certaines circonstances seulement est prévu en France, en Autriche (article 44, alinéa 3, de la Loi fédérale constitutionnelle du 10 novembre 1920), en Espagne (art. 167, alinéa 3, de la Constitution du 29 décembre 1978), et en Italie (article 138, alinéas 2 et 3, de la Constitution du 27 décembre 1947).

${ }^{170}$ Un mécanisme de dissolution automatique des Chambres est prévu au $\$ 88$ de la Constitution danoise du 5 juin 1953 et à l'article 137 , alinéa 3 , de la nouvelle Constitution néerlandaise du 17 février 1983. - Un mécanisme de dissolution automatique dans certains cas seulement est prévu par l'article 168, alinéa $1^{\text {er }}$ et 2, de la Constitution espagnole du 29 décembre 1978.

${ }^{171}$ Tel est le cas en Italie, en Allemagne et en Autriche. - En Italie, l'article 138 de la Constitution du 27 décembre 1947 exige pour la révision de la Constitution un double vote dans chaque Chambre, espacé de trois mois, mais à la majorité ordinaire seulement. En Allemagne, l'article 79, alinéa 2, de la Loi fondamentale du 23 mai 1949 requiert dans chaque chambre un seul vote à la majorité des deux tiers. Enfin, en Autriche, l'article 44, alinéas $1^{\text {er }}$ et 2, de la Loi fédérale constitutionnelle du 10 novembre 1920 exige un vote à la majorité des deux tiers au seul Nationalrat. Ce n'est que si les compétences des Länder sont concernées qu'un vote aux mêmes conditions est par ailleurs organisé au Bundesrat. Un tiers des membres de l'une des deux Chambres peut cependant demander la tenue d'un référendum national.

${ }^{172}$ Tel est notamment le cas de la Grèce, pays qui en raison de son passé dictatorial contient une disposition rendant impossible toute révision de la Constitution pendant un délai de cinq ans à compter de la dernière révision, et cela même si la seconde révision concerne une disposition autre que celle ayant fait l'objet de la première révision (voy. l'article 110, alinéa 6, de la Constitution du 9 juin 1975). - Un mécanisme similaire existe au Portugal, pays accusant également un passé dictatorial : l'article 284 de la Constitution du 2 avril 1976 contient le même principe et la même période de cinq ans mais n'instaure pas une interdiction absolue de révision car un amendement constitutionnel demeure possible avant l'expiration du délai fixé, mais requiert alors une majorité des quatre cinquièmes.

${ }^{173}$ La révision de la Constitution fédérale des Etats-Unis d'Amérique du 17 septembre 1787, requiert, conformément à son article V, l'approbation du projet de révision les par trois quarts des Etats fédérés. Cette procédure ne convient pas en Belgique en raison du fait qu'elle fait participer les entités fédérées au processus de révision (voy. supra, $\mathrm{n}^{\circ} 24$ et note 97), et même si l'on acceptait ce principe, il serait inimaginable en Belgique d'exiger l'assentiment des trois quarts d'entre elles seulement. Par ailleurs, il convient de ré-insister sur la lourdeur procédurale qu'entraîne la 


\section{VI - CONCLUSIONS}

40. - Dans le présent exposé, nous n'avons pas traité de l'opportunitité d'une révision de l'article 195 de la Constitution belge mais sommes parti du présupposé que la décision de modifier sa version actuelle moribonde et ouverte à révision - soit politiquement acquise. A cet égard, notre conclusion est double.

\section{A - DANS LA DETERMINATION DU NOUVEAU LIBELLE DE L'ARTICLE 195, LE CONSTITUANT DISPOSE D'UN POUVOIR DECISIONNEL REDUIT MAIS REEL}

41. - D'abord, on peut constater que la marge de manœuvre réelle du Pouvoir constituant pour la révision de l'article 195 est assez peu étendue ; à l'origine de cette situation se trouve un certain nombre de contraintes factuelles, tant générales que spécifiquement belges (IV).

Un pouvoir décisionnel réduit n'équivaut cependant pas à un pouvoir décisionnel inexistant (V): ainsi, il importera de trancher la question du maintien ou non d'un mécanisme unique de révision, il conviendra d'examiner quels avantages présente encore à l'heure actuelle la technique de la déclaration de révision, il faudra analyser en détail le problème des mécanismes de temporisation et il sera nécessaire de déterminer la base précise pour le calcul des majorités.

Un pouvoir décisionnel réduit n'équivaut pas non plus à un pouvoir décisionnel sans effet : ainsi, il convient d'exprimer l'espoir de pouvoir assister, grâce à la révision de l'article 195, à une amélioration qualitative et une application effective du texte de la Loi fondamentale belge. L'amélioration qualitative pourrait notamment se traduire par une réécriture du vétuste titre II $^{176}$, la suppression d'un bon nombre de dispositions obsolètes ${ }^{177}$ et - qui sait ? - une réglementation constitutionnelle des rapports entre droit interne et international qui fait cruellement défaut depuis des décennies ${ }^{178}$. L'application effective pourrait, quant à elle, se manifester par une renaissance des articles 46 et 96 de la Constitution, dispositions jusqu'ores reléguées à un rôle de simples figurants.

\footnotetext{
participation des entités fédérées à la procédure de révision : la meilleure illustration en est le XXVII ${ }^{\mathrm{e}}$ amendement de la Constitution des EtatsUnis, document proposé en 1789 et dont l'approbation par les trois quarts des Etats fédérés n'a pu être réalisée que 203 ans plus tard, en 1992.

${ }^{174}$ Le $\S 15$ du Chapitre 8 de la Constitution suédoise du $1^{\mathrm{er}}$ janvier 1975 prévoit le mécanisme suivant : une modification de la Constitution nécessite un double vote du même texte. Les deux votes doivent être étalés sur deux législatures différentes. Par ailleurs, un délai minimum de neuf mois doit s'être écoulé entre le premier et le second scrutin. Il peut cependant être dérogé à cette dernière condition par un vote, à la majorité draconienne des cinq sixièmes, au sein de la Commission parlementaire chargée de la Révision de la Constitution. - L'inconvénient de la procédure suédoise est cependant de confier le vote sur la mise en œuvre de la procédure dérogatoire non pas au Parlement réuni en séance plénière mais à une simple Commission parlementaire. Par ailleurs, le $\S 15$ ne prévoit aucune condition particulière de majorité pour le vote de la révision de la Constitution.

${ }^{175}$ Le $§ 73$ de la Constitution finlandaise du 11 juin 1999 prévoit la procédure suivante : une révision de la Constitution requiert un double vote du même texte, le second scrutin devant se faire à la majorité des deux tiers. En principe, les deux votes ne peuvent avoir lieu au cours de la même législature ; en outre, le second vote ne peut intervenir avant l'écoulement d'un délai de douze mois à compter des élections. Cependant, le second vote peut immédiatement suivre le premier si tel est le souhait d'une majorité des cinq septièmes des membres du Parlement réuni en séance plénière.

${ }^{176}$ A ce sujet, voy. notamment S. van Drooghenbroeck, « Pour une mise à jour du Droit constitutionnel belge des libertés publiques et des droits de l'homme (...) », Administration publique trimestrielle, 2001, p. 130-153, spécialement p. 142-152.

${ }^{177}$ Voy. notamment K. Rimanque, « Overbodig geworden bepalingen in de Grondwet - vroeger en nu », Publiek recht, ruim bekeken, Opstellen aangeboden aan Prof. J. Gijssels, Anvers, Maklu, 1994, p. 241-253.

${ }^{178}$ Et ce malgré la circonstance que sept déclarations de révision $(1965,1968,1978,1981,1987,1991$ et 1995) ont été votées en ce sens. - Sur le sujet des rapports entre droit national et international, voy. par ailleurs les contributions de W. Ganshof van der Meersch, « Réflexions sur le droit international et la révision de la Constitution », Journal des Tribunaux, 1968, p. 485-496, l'ouvrage collectif intitulé L'effet direct en droit belge des traités internationaux en général et des instruments internationaux relatifs aux droits de l'homme en particulier, Bruxelles, Bruylant et Editions de l'Université de Bruxelles, 1981, 354 pages, F. Dumon, « Les rapports entre le droit constitutionnel et le droit international », Le nouveau droit constitutionnel, précité, p. 205-223, J. Velu, "Contrôle de constitutionnalité et contrôle de compatibilité avec les traités », Journal des Tribunaux, 1992, p. 729-741 et p. 749-761, A. Beirlaen, « Omtrent de verhouding tussen het internationaal en het Belgisch recht », Présence du droit public et des droits de l'homme, Mélanges offerts à Jacques Velu, tome 1, Bruxelles, Bruylant, 1992, p. 141-151, H. Bribosia, « Applicabilité directe et primauté des traités internationaux et du droit communautaire », Revue belge de Droit international, 1996, p. 33-89, et J.-S. Jamart, « Observations sur l'argumentation : la primauté du droit international », Revue belge de Droit constitutionnel, 1999, p. 109-136.
} 


\section{B - L'ARTICLE 195 CONTINUERA A AVOIR UNE INCIDENCE CAPITALE SUR LA STABILITE NORMATIVE DE LA CONSTITUTION ET PLUS GENERALEMENT SUR CELLE DE L'ORDRE JURIDIQUE BELGE}

42. - Ensuite, les développements qui précèdent font ressortir l'importance particulière du concept de rigidité de la Constitution. C'est cette dernière notion qui occupe une place centrale dans le maintien de l'autre objectif, tout aussi essentiel, de stabilité de la Constitution.

L'article 195 - l'actuel comme son éventuelle version future - demeure un facteur tout à fait déterminant pour la stabilité de la Constitution. Or, dans un Etat aussi polarisé et centrifuge que la Belgique, il n'est nul besoin de souligner que de la stabilité de la Constitution dépend celle du Royaume. Quand Jürgen Habermas affirme que «[d]es considérations de stabilité ne peuvent pas tenir lieu de réflexions normatives ${ }^{179}$, il faut bien convenir qu'il a fondamentalement tort. Dans tout Etat, et a fortiori en Belgique, la stabilité constitutionnelle constitue non seulement la condition sine qua non de la sécurité juridique mais représente également une valeur en soi, valable comme argument normatif : si l'on veut vraiment éviter que les textes fondateurs de notre Etat ne soient considérés comme des simples « documents précaires ${ }^{180}$, il faut les préserver contre toute forme de souplesse destructrice.

A cet égard l'article 195 a fait ses preuves, malgré ses imperfections. Sa modification, à la supposer acquise, doit dès lors se faire de manière circonspecte, en préservant sa rigidité. C'est dans des circonstances aussi importantes que celles-ci qu'il convient de rappeler les mots de Portalis, qui, à propos de changements législatifs, insistait : "Il faut changer quand la plus funeste de toutes les innovations serait, pour ainsi dire, de ne pas innover. Tout ce qui est ancien a été nouveau. L'essentiel est d'imprimer aux institutions nouvelles le caractère de permanence et de stabilité qui puisse leur garantir le droit de devenir anciennes. » ${ }^{181}$

Ce qui est vrai pour les lois, l'est d'autant plus pour la Constitution. On le dit trop peu.

Mai 2003

[Contribution publiée dans la Revue française de droit constitutionnel, 2003, 279-308]

\footnotetext{
${ }^{179}$ J. Habermas, Ecrits politiques, Paris, Cerf, 1990, p. 257, cité par H. Dumont, « La réforme de 1993 et la question du référendum constituant », précité, p. 104

${ }^{180}$ J. Rivero, «Fin d'un absolutisme », Pouvoirs, n 13 (1980), p. 7.

${ }^{181}$ J.E.M. Portalis, Exposé des motifs de la loi du 30 Ventôse an XII, n ${ }^{2}$, cité par J.-G. Locré, Esprit du Code Napoléon, tiré de la discussion, ou conférence historique, analytique et raisonnée du projet du Code civil, des observations des tribunaux, des procès-verbaux du conseil d'état, des observations du tribunat, des exposés de motifs, des rapports et discours etc., etc.[sic]; dédié à S.M. l'Empereur et Roi, tome 1, Paris, Imprimerie impériale, 1805, p. 199 (C'est nous qui soulignons.).
} 